
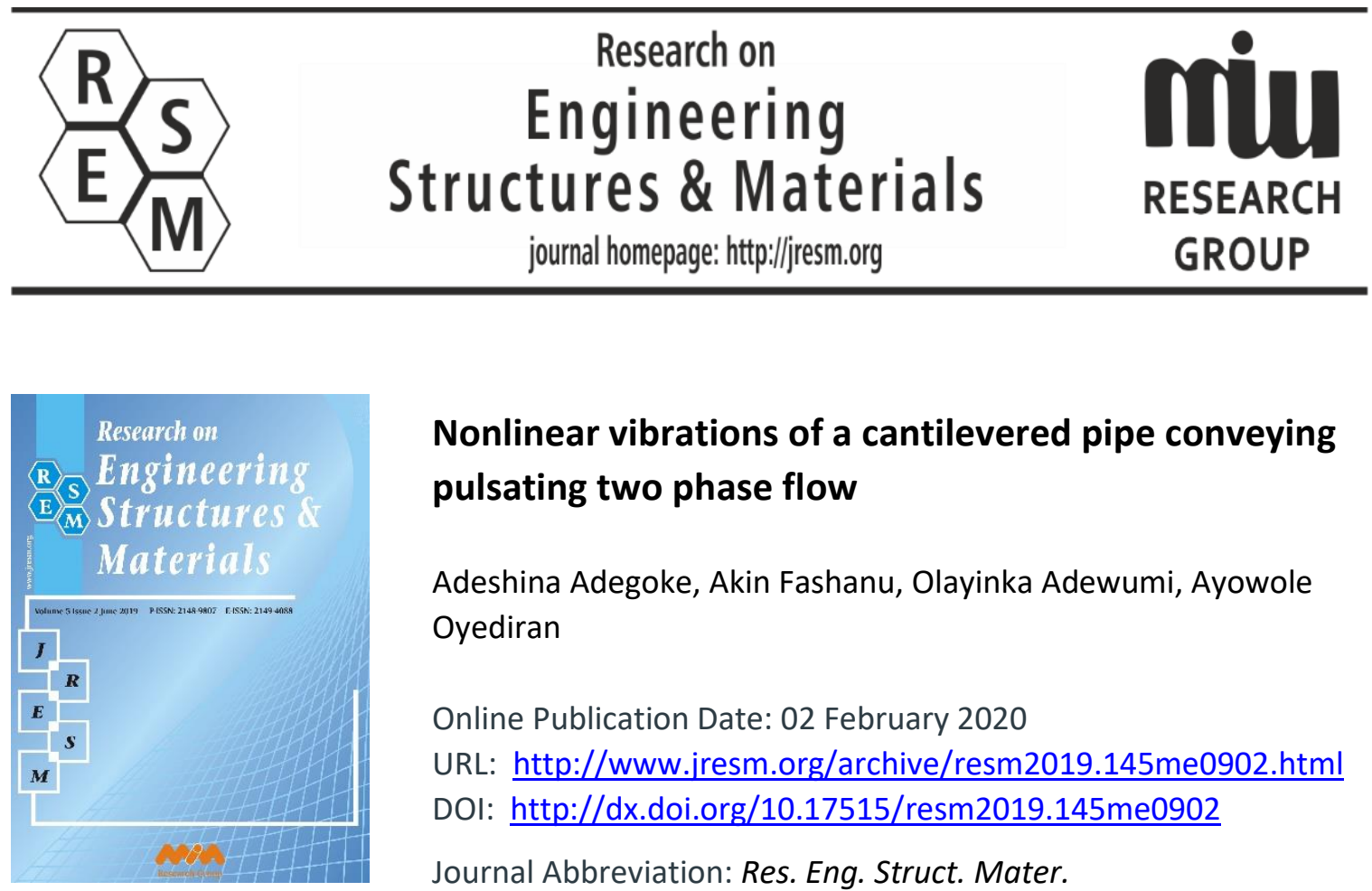

\title{
Nonlinear vibrations of a cantilevered pipe conveying pulsating two phase flow
}
Adeshina Adegoke, Akin Fashanu, Olayinka Adewumi, Ayowole Oyediran

Online Publication Date: 02 February 2020

URL: http://www.jresm.org/archive/resm2019.145me0902.html

DOI: http://dx.doi.org/10.17515/resm2019.145me0902

Journal Abbreviation: Res. Eng. Struct. Mater.

\section{To cite this article}

Adegoke A, Fashanı A, Adewumi O, Oyediran A. Nonlinear vibrations of a cantilevered pipe conveying pulsating two phase flowield response prediction of unsymmetrically reinforced concrete rectangular beams. Res. Eng. Struct. Mater., 2020; 6(3): 207-227.

\section{Disclaimer}

All the opinions and statements expressed in the papers are on the responsibility of author(s) and are not to be regarded as those of the journal of Research on Engineering Structures and Materials (RESM) organization or related parties. The publishers make no warranty, explicit or implied, or make any representation with respect to the contents of any article will be complete or accurate or up to date. The accuracy of any instructions, equations, or other information should be independently verified. The publisher and related parties shall not be liable for any loss, actions, claims, proceedings, demand or costs or damages whatsoever or howsoever caused arising directly or indirectly in connection with use of the information given in the journal or related means.

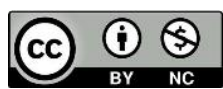
Published articles are freely available to users under the terms of Creative Commons Attribution - NonCommercial 4.0 International Public License, as currently displayed at here (the "CC BY - NC"). 


\title{
Research on Engineering Structures \& Materials
}

journal homepage: http://jresm.org

Research Article

\section{Nonlinear vibrations of a cantilevered pipe conveying pulsating two phase flow}

\author{
Adeshina Adegoke1,a, Akin Fashanu²,b, Olayinka Adewumi1,c, Ayowole Oyediran 1,d* \\ ${ }^{1}$ Department of Mechanical Engineering, University of Lagos, Akoka-Yaba, Lagos, Nigeria. \\ ${ }^{2}$ Department of Systems Engineering, University of Lagos, Akoka-Yaba, Lagos, Nigeria.
}

\begin{tabular}{|c|c|}
\hline Article Info & Abstract \\
\hline $\begin{array}{l}\text { Article history: } \\
\text { Received } 02 \text { Sep } 2019 \\
\text { Revised 09 Dec } 2019 \\
\text { Accepted } 26 \text { Dec } 2019 \\
\end{array}$ & $\begin{array}{l}\text { This work studied the nonlinear transverse vibrations of a cantilevered pipe } \\
\text { conveying pulsatile two-phase flow. Internal flow induced parametric resonance } \\
\text { is expected because of the time varying velocity of the conveyed fluid. This } \\
\text { unsteady behaviour of the conveyed two-phase flow is considered in the } \\
\text { governing equation as time dependent individual velocities with the }\end{array}$ \\
\hline Keywords: & $\begin{array}{l}\text { harmonically varying components fluctuating about the constant mean } \\
\text { velocities. Method of multiple scales analysis is adopted to study the nonlinear } \\
\text { parametric resonance of dynamics of the cantilevered pipe. Contrary to the }\end{array}$ \\
\hline Nonlinear dynamics; & dynamics of pulsating single-phase flow, the assessment shows that if the \\
\hline Transverse vibration; & $\begin{array}{l}\text { frequencies of pulsation of the two phases are close, both can resonate with the } \\
\text { pipe's transverse or axial frequencies together and both can also independently }\end{array}$ \\
\hline flow; Parametric & esonate with the pipe's transverse or axial frequencies distinctively. For the \\
\hline resonance; & lanar dvnamics when only transverse frequencies are resonated in the abs \\
\hline Cantilevered pipe & $\begin{array}{l}\text { of internal resonance, numerical results show that the system exhibits softening } \\
\text { nonlinear behavior. At post critical flow conditions, the system oscillates } \\
\text { between subcritical and supercritical pitchfork bifurcation to simulate the } \\
\text { nonlinear Mathieu's equation. However, in the presence of internal resonance, a } \\
\text { nonlinear anti-resonance property is developed. Hence, the overall dynamics is } \\
\text { quasi-periodic. }\end{array}$ \\
\hline
\end{tabular}

(C) 2020 MIM Research Group. All rights reserved.

\section{Introduction}

Regardless of a floater concept for a deepwater field development, there is always a need of riser system connecting the floater at surface and subsea facility on the seabed as explained by Ruxin et al [1]. However, these riser systems are known to be of different variants. One of which is the single hybrid riser (SHR) system, a free-standing riser pipe decoupled from the floater with the aid of a flexible jumper. Chung et al [2] shows that the rich dynamics of cantilever pipes can be adopted as a paradigm for demonstrating the complex dynamic characteristics of this type of riser pipes in deep ocean mining.

As a result of barometric effects, thermal effects and the composition of the hydrocarbon compounds, these riser pipes sometimes convey fluid that can be a single phase liquid or a single phase gas or a multiple-phase mixture. It is a known fact that dynamics of multiphase flow are much more complex when compared to that of single-phase flows. A very common multiphase flow is the gas and liquid two phase flow. The flow behaviour of a gas and liquid two phase flow brings about the complex interaction of numerous variables which complicates the engineering of the conduits that conveys them.

\footnotetext{
${ }^{*}$ Corresponding author: ayooyediran@hotmail.com

a orcid.org/0000-0003-0678-7951; b orcid.org/0000-0003-0958-1641; c orcid.org/0000-0002-3545-6679;

d orcid.org/0000-0003-3482-9063

DOI: http://dx.doi.org/10.17515/resm2019.145me0902
}

Res. Eng. Struct. Mat. Vol. 6 Iss. 3 (2020) 207-227 
The fluid elastic instability of pipes conveying fluid is mainly due to the unstable vibration caused by the fluid flow, when the flow velocity surpasses a critical value and also due to harmonically perturbed fluid flow (Pulsating Flows). Nevertheless, the effect of the dynamics of pipes conveying fluids has been widely studied by many authors, with most of the studies focusing on the linear and nonlinear dynamics of pipes conveying steady flow as seen in [3-7]. However, it was highlighted by Ibrahim [8] that an obvious effect of pulsating fluid flow is that it forces the pipeline into sustained vibrations under parametric resonance conditions. Forlornly, flow conveyed by pipes are rarely steady. Luczko and Czerwinski. [9] explains that components of hydraulic systems such as pumps, hydraulic engines, fast valves opening and closing, timely injection of inhibitors, elbows, orifices, flow through reducers and many other sources of perturbations often generate pulsation of fluid flow.

All the same, the studies on the linear and nonlinear dynamics of pipes conveying pulsating flow cannot be seen as new. Earlier works can be dated back to more than four decades for various end conditions. Ginsberg [10] pioneered the work considering pinned-pinned pipes, then Paidoussis and Issid [11] for cantilevered pipes and Paidoussis and Sundararajan [12] for clamped-clamped pipes. Sequel to these early studies, the nonlinear dynamics of the subject captivated the minds of many curious researchers which resulted to various publications; notable among these, are the works of Semler and Paidoussis [13] on the nonlinear analysis of parametric resonance of a planar fluid-conveying cantilevered pipe using four different methods; Centre manifold theory, perturbation method, finite difference method and Increment harmonic balance method to resolve the equation and also comparing the theoretical results with experiments. Namachchivaya and Tien [14] on the nonlinear behaviour of supported pipes conveying pulsating fluid examined the problem in the vicinity of subharmonic and combination resonance using the method of averaging. Pranda and Kar [15] on the nonlinear dynamics of a pipe conveying pulsating flow with a combination, principal parametric and internal resonance, adopting the method of multiple scale to resolve the transverse vibrations and stability of a hingedhinged pipe, Liangqiang et al [16] adopted the multiple scale and normal form theory to determine the expressions for the critical bifurcations leading to incipient and secondary bifurcations for the transverse vibration of a hinged-hinged pipe, $\mathrm{Oz}$ and Boyaci [17] on the transverse vibrations of tensioned pipes conveying fluid with time-dependent velocity, adopting the method of multiple scale to analytically determine the stability boundaries on the system, also considered the cases of fixed-fixed end and fixed-sliding end conditions with a varnishing flexural stiffness. $\mathrm{Oz}$ [18] on the analysis of nonlinear vibrations and stability analysis of tensioned pipe conveying fluid with variable velocity studied the pipe with fixed supports and immovable end conditions with the flexural stiffness neglected. Singh and Mallik [19] used the wave propagation approach to determine the parametric instability regions of a periodically supported pipe conveying fluid. Yoshizawa et al. [20] analytically and experimentally studied the lateral vibration of a flexible clamped-hinged pipe parametrically excited by a fluctuating harmonic internal flow, it was shown that as the amplitude of the pipe deflection grows, the nonlinearity of the fluid force acting on the pipe limits the growth, which results in a steady-state vibration.

On the instability behaviour of pipes conveying multiphase flow, Miwa et al. [21] did a review of the extent of existing work on two-phase flow induced vibrations, stating that there exist very few researches on the instability behaviour of pipes due to internal twophase flow. Some of the few existing publications on this subject are the works of Monette and Pettigrew [22] which experimentally and theoretically reveals the relationship between the void fraction and the dynamics of the pipe for a two-phase liquid-gas flow. Adegoke and Oyediran [23] showed that the attainment of the critical velocities in the axial and transverse direction are delayed for a cantilever pipe conveying two phase flow 
compared to when the pipe is conveying single phase flow. In addition, the critical velocity is observed to be increasing as the void fraction of the two-phase flow increases. Adegoke and Oyediran [24] studied the nonlinear vibrations of top-tensioned cantilevered pipes conveying pressurized steady two-phase flow under thermal loading. The multiple-scale assessment reveals that at some frequencies the system is uncoupled, while at other frequencies a 1:2 coupling exists between the axial and the transverse frequencies of the pipe. Wang et al. [25] adapted the linear equation of transverse motion for single phase flow to account for the two phases and resolved the modified equation using finite element method; experiments were also performed to measure the characteristic parameters of the hydrodynamic slugs and the dynamics response of the pipe. Ortiz-Vidal et al [26] on the theoretical study of the dynamic behaviour of pipes conveying Gas-Liquid flow, also adapted the linear equation of transverse motion for single phase flow to account for the two phases and resolved the modified equation using Galerkin's method for a pipe clamped at both ends.

As seen in the review of literature, most of the existing publications focused on pipes conveying pulsating single phase flow while most of the analytical works on the nonlinear problem did not consider the internal coupling between the axial and the transverse vibrations.Also, some of the publications neglected the flexural stiffness and modelled the pipe as a string with internal fluid flow. On the other hand, recent publications on two phase flow worked on establishing the governing equations and the resolution of the linear dynamics. While the publication on the nonlinear dynamics of pipes conveying two phase flow considered the flow to be steady, neglecting the unsteady velocity term while assuming that the flow exhibits a constant flow mixture velocity. However, to the best of our knowledge, there seems to be some gaps on the study of the nonlinear dynamics of cantilever pipes conveying pulsating two phase fluids. This present study investigates the coupled axial and transverse vibrations of a cantilever pipe conveying pulsating two phase flow. Approximate analytical approach will be used to resolve the governing equations by imposing the method of multiple scales perturbation technique directly to the systems equations (direct-perturbation method).

\section{Problem formulation and modeling}

In this work, we considered and assumed a system of cantilevered cylindrical pipe of length (L), with cross-sectional area (A), mass per unit length (m), flexural rigidity (EI), and conveying multiphase flow. 1. Plug flow velocity profile. 2. The pipe is modelled as EulerBernoulli beam. 3. The motion is planar. 4. Pipe deflections are large, but strains are small. 5. Pipe centerline is extensible.

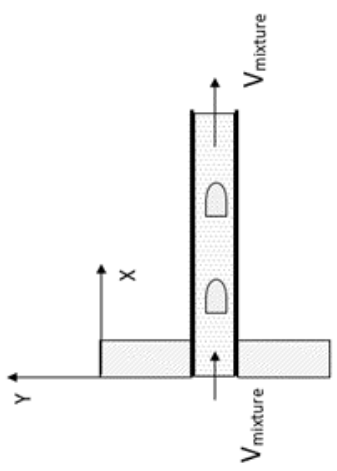

Fig. 1 System's Schematic 


\subsection{Equation of motion}

Adopting the equations of motion of an extensible pipe conveying multi-phase flow as obtained by [24]

$$
\begin{aligned}
\left(m+\sum_{j=1}^{n} M_{j}\right) \ddot{u}+ & \sum_{j=1}^{n} \mathrm{M}_{j} \dot{U}_{J}+\sum_{j=1}^{n} 2 M_{j} U_{j} \dot{u}^{\prime}+\sum_{j=1}^{n} M_{j} U_{j}^{2} u^{\prime \prime}+\sum_{j=1}^{n} M_{j} \dot{U}_{j} u^{\prime}-E A u^{\prime \prime} \\
& -E I\left(v^{\prime \prime \prime \prime} v^{\prime}+v^{\prime \prime} v^{\prime \prime \prime}\right)+\left(T_{0}-P-E A(\alpha \Delta T)-E A\right) v^{\prime} v^{\prime \prime} \\
& -\left(T_{0}-P-E A(\alpha \Delta T)\right)^{\prime}+\left(m+\sum_{j=1}^{n} M_{j}\right) g=0, \\
\left(m+\sum_{j=1}^{n} M_{j}\right) \ddot{v}+ & \sum_{j=1}^{n} 2 M_{j} U_{j} \dot{v}^{\prime}+\sum_{j=1}^{n} M_{j} U_{j}^{2} v^{\prime \prime}-\sum_{j=1}^{n} a M_{j} U_{j}^{2} v^{\prime \prime}+\sum_{j=1}^{n} M_{j} \dot{U}_{j} v^{\prime} \\
& +E I v^{\prime \prime \prime \prime}-\left(T_{0}-P-E A(\alpha \Delta T)\right) v^{\prime \prime} \\
& -E I\left(3 u^{\prime \prime \prime} v^{\prime \prime}+4 v^{\prime \prime \prime} u^{\prime \prime}+2 u^{\prime} v^{\prime \prime \prime \prime}+v^{\prime} u^{\prime \prime \prime \prime}+2 v^{\prime 2} v^{\prime \prime \prime \prime}\right. \\
& \left.+8 v^{\prime} v^{\prime \prime} v^{\prime \prime \prime}+2 v^{\prime \prime 3}\right) \\
& +\left(T_{0}-P-E A(\alpha \Delta T)-E A\right)\left(u^{\prime} v^{\prime \prime}+v^{\prime} u^{\prime \prime}+\frac{3}{2} v^{\prime 2} v^{\prime \prime}\right)=0
\end{aligned}
$$

The associated boundary conditions are:

$$
v(0)=v^{\prime}(0), v^{\prime \prime}(L)=v^{\prime \prime \prime}(L)=0 \text { and } u(0)=u^{\prime}(L)=0
$$

Where $x$ is the longitudinal axis, $v$ is the transverse deflection, $u$ is the axial deflection, $n$ is the number of phases which is 2 in this study. In the fluid, $M_{j}$ is the mass of the $j^{\text {th }}$ phase and $U_{j}$ is its corresponding speed. $m$ is the mass of the pipe, $T_{o}$ is tension, $P$ is the pressure, $\alpha$ is the thermal expansivity term, $\Delta T$ relates to the temperature difference and $a$ relates to the Poisson ration $(r)$ as $a=1-2 r$.

Using these dimensionless quantities and notations:

$$
\begin{aligned}
& \bar{u}=\frac{u}{L}, \quad \bar{v}=\frac{v}{L}, \quad \bar{t}=\left[\frac{E I}{\sum M_{j}+m}\right]^{1 / 2} \frac{t}{L^{2}}, \quad \bar{U}_{j}=\left[\frac{M_{j}}{E I}\right]^{1 / 2} U L, \gamma=\frac{\sum M_{j}+m}{E I} L^{3} g, \\
& \Psi_{j}=\frac{M_{j}}{\sum M_{j}}, \Pi_{0}=\frac{T_{o} L^{2}}{E I}, \Pi_{1}=\frac{E A L^{2}}{E I}, \Pi_{2}=\frac{P L^{2}}{E I} \cdot \beta_{j}=\frac{M_{j}}{\sum M_{j}+m},
\end{aligned}
$$

Notations:

$C 11=\sqrt{\Psi_{1}} \sqrt{\beta_{1}}, C 12=\sqrt{\Psi_{2}} \sqrt{\beta_{2}}, C 21=2 \sqrt{\Psi_{1}} \sqrt{\beta_{1}}, C 22=2 \sqrt{\Psi_{2}} \sqrt{\beta_{2}}, C 31=\Psi_{1}$, $C 32=\Psi_{2}, C 5=\Pi_{1}, C 6=\left(\Pi_{0}-\Pi_{2}-\Pi_{1}(\alpha \Delta T)-\Pi_{1}\right), C 7=\Pi_{0}-\Pi_{2}-\Pi_{1}(\alpha \Delta T)$.

The equation is reduced to that of a two-phase flow as:

$$
\begin{aligned}
& \ddot{\bar{u}}+\dot{\overline{U_{1}}} C 11+\dot{\bar{U}_{2}} C 12+\bar{U}_{1} C 21 \dot{\bar{u}}^{\prime}+\bar{U}_{2} C 22 \dot{\bar{u}}^{\prime}+C 31{\overline{U_{1}}}^{2} \bar{u}^{\prime \prime}+C 32{\overline{U_{2}}}^{2} \bar{u}^{\prime \prime}+ \\
& \dot{U_{1}} C 11 \bar{u}^{\prime}+\overline{U_{2}} C 12 \bar{u}^{\prime}-C 5 \bar{u}^{\prime \prime}-\left(\bar{v}^{\prime \prime \prime \prime} \bar{v}^{\prime}+\bar{v}^{\prime \prime} \bar{v}^{\prime \prime \prime}\right)+C 6 \bar{v}^{\prime} \bar{v}^{\prime \prime}-C 7^{\prime}+\gamma=0
\end{aligned}
$$




$$
\begin{aligned}
& \ddot{\bar{v}}+\bar{U}_{1} C 21 \dot{\bar{v}}^{\prime}+\bar{U}_{2} C 22 \dot{\bar{v}}^{\prime}+C 31{\overline{U_{1}}}^{2} \bar{v}^{\prime \prime}+C 32{\overline{U_{2}}}^{2} \bar{v}^{\prime \prime}-a C 31{\overline{U_{1}}}^{2} \bar{v}^{\prime \prime}-a C 32{\overline{U_{2}}}^{2} \bar{v}^{\prime \prime}+ \\
& \dot{U_{1}} C 11 \bar{v}^{\prime}+{\dot{U_{2}}}_{2} C 12 \bar{v}^{\prime}-C 8 \bar{v}^{\prime \prime}+\bar{v}^{\prime \prime \prime \prime}-\left(3 \bar{u}^{\prime \prime \prime} \bar{v}^{\prime \prime}+4 \bar{v}^{\prime \prime \prime} \bar{u}^{\prime \prime}+2 \bar{u}^{\prime} \bar{v}^{\prime \prime \prime \prime}+\bar{v}^{\prime} \bar{u}^{\prime \prime \prime \prime}+\right. \\
& \left.2 \bar{v}^{\prime 2} \bar{v}^{\prime \prime \prime \prime}+8 \bar{v}^{\prime} \bar{v}^{\prime \prime} \bar{v}^{\prime \prime \prime}+2 \bar{v}^{\prime \prime}\right)+C 6\left(\bar{u}^{\prime} \bar{v}^{\prime \prime}+\bar{v}^{\prime} \bar{u}^{\prime \prime}+\frac{3}{2} \bar{v}^{\prime 2} \bar{v}^{\prime \prime}\right)=0
\end{aligned}
$$

For the purpose of this analysis, two novel driving functions $U_{1}$ and $U_{2}$ are introduced to pulsate the two phases such that their velocities fluctuate harmonically at frequencies $\left(\Omega_{1}\right.$ and $\left.\Omega_{2}\right)$ about constant mean values $\left(U_{0_{1}}\right.$ and $\left.U_{0_{2}}\right)$. This differs from the steady velocities considered in [24]. Precisely, the driving functions are expressed as;

$$
U_{1}=U_{0_{1}}\left(1+\mu_{1} \sin \left(\Omega_{1} \mathrm{~T}_{0}\right)\right) \text { and } U_{2}=U_{0_{2}}\left(1+\mu_{2} \sin \left(\Omega_{2} \mathrm{~T}_{0}\right)\right)
$$

For the two-phase flow, the empirical relationship is obtained by expressing the component's velocities in terms of the superficial velocities as:

$$
V_{g}=U_{g} v_{f}, \quad V_{l}=U_{l}\left(1-v_{f}\right)
$$

Where $U_{g}$ and $U_{l}$ are the superficial flow velocities.

Adopting the Chisholm empirical relations as presented in [27],

Void fraction:

$$
v_{f}=\left[1+\sqrt{1-\mathrm{x}\left(1-\frac{\rho_{l}}{\rho_{g}}\right)}\left(\frac{1-\mathrm{x}}{\mathrm{x}}\right)\left(\frac{\rho_{g}}{\rho_{l}}\right)\right]^{-1}=\frac{\text { Volume of gas }}{\text { Volume of gas+Volume of Liquid }}
$$

Slip Ratio: $S=\frac{V_{g}}{V_{l}}=\left[1-\mathrm{x}\left(1-\frac{\rho_{l}}{\rho_{g}}\right)\right]^{1 / 2}$

The vapour quality: $(\mathrm{x})$

The densities of the liquid and gas phases respectively: $\left(\rho_{l}\right.$ and $\left.\rho_{g}\right)$

$$
\text { Mixture Velocity: } V_{T}=U_{g} v_{f}+U_{l}\left(1-v_{f}\right)
$$

Individual Velocities:

$$
V_{l}=\frac{V_{T}}{S+1}, V_{g}=\frac{S V_{T}}{S+1}
$$

For various void fractions $(0.1,0.3$, and 0.5$)$ and a series of mixture velocities, the corresponding slip ratio and individual velocities are estimated and used for calculations.

\section{Method of Solution}

We seek an approximate solution for $\bar{u}$ and $\bar{v}$ in the form:

$$
\begin{aligned}
& \bar{u}=\bar{u}_{0}\left(T_{0}, T_{1}\right)+\varepsilon \bar{u}_{1}\left(T_{0}, T_{1}\right)+\varepsilon^{2} \bar{u}_{2}\left(T_{0}, T_{1}\right)+O(\varepsilon) \\
& \bar{v}=\bar{v}_{0}\left(T_{0}, T_{1}\right)+\varepsilon \bar{v}_{1}\left(T_{0}, T_{1}\right)+\varepsilon^{2} \bar{v}_{2}\left(T_{0}, T_{1}\right)+O(\varepsilon)
\end{aligned}
$$

For this purpose, two time scales are needed $T_{0}=t$ and $T_{1}=\varepsilon t$. Where $\varepsilon$ is used as a bookkeeping parameter. Perturbing the non-dimensional form of the governing equations and the pulsation of the phases, equations $(14,15$ and 16$)$ are realized;

$$
\begin{aligned}
& \ddot{\bar{u}}+\dot{\overline{U_{1}}} C 11+\dot{\overline{U_{2}}} C 12+\bar{U}_{1} C 21 \dot{\bar{u}}^{\prime}+\bar{U}_{2} C 22 \dot{\bar{u}}^{\prime}+C 31{\overline{U_{1}}}^{2} \bar{u}^{\prime \prime}+C 32{\overline{U_{2}}}^{2} \bar{u}^{\prime \prime}+ \\
& \dot{U_{1}} C 11 \bar{u}^{\prime}+\dot{U_{2}} C 12 \bar{u}^{\prime}-C 5 \bar{u}^{\prime \prime}+\varepsilon\left(-\left(\bar{v}^{\prime \prime \prime \prime} \bar{v}^{\prime}+\bar{v}^{\prime \prime} \bar{v}^{\prime \prime \prime}\right)+C 6 \bar{v}^{\prime} \bar{v}^{\prime \prime}-C 7^{\prime}+\gamma\right)=0
\end{aligned}
$$




$$
\begin{aligned}
& \ddot{\bar{v}}+\bar{U}_{1} C 21 \dot{\bar{v}}^{\prime}+\bar{U}_{2} C 22 \dot{\bar{v}}^{\prime}+C 31{\overline{U_{1}}}^{2} \bar{v}^{\prime \prime}+C 32{\overline{U_{2}}}^{2} \bar{v}^{\prime \prime}-a C 31{\overline{U_{1}}}^{2} \bar{v}^{\prime \prime}- \\
& a C 32{\overline{U_{2}}}^{2} \bar{v}^{\prime \prime}+\dot{\bar{U}_{1}} C 11 \bar{v}^{\prime}+\dot{U_{2}} C 12 \bar{v}^{\prime}-C 7 \bar{v}^{\prime \prime}+\bar{v}^{\prime \prime \prime \prime}+\varepsilon\left(-\left(3 \bar{u}^{\prime \prime \prime} \bar{v}^{\prime \prime}+4 \bar{v}^{\prime \prime \prime} \bar{u}^{\prime \prime}+\right.\right. \\
& \left.2 \bar{u}^{\prime} \bar{v}^{\prime \prime \prime \prime}+\bar{v}^{\prime} \bar{u}^{\prime \prime \prime \prime}+2 \bar{v}^{\prime 2} \bar{v}^{\prime \prime \prime \prime}+8 \bar{v}^{\prime} \bar{v}^{\prime \prime} \bar{v}^{\prime \prime \prime}+2 \bar{v}^{\prime \prime}\right)+C 6\left(\bar{u}^{\prime} \bar{v}^{\prime \prime}+\bar{v}^{\prime} \bar{u}^{\prime \prime}+\right. \\
& \left.\left.\frac{3}{2} \bar{v}^{\prime 2} \bar{v}^{\prime \prime}\right)\right)=0 \\
& \overline{U_{1}}=\overline{U_{1}}\left(1+\varepsilon \mu_{1} \sin \left(\Omega_{1} \mathrm{~T}_{0}\right)\right) \text { and } \overline{U_{2}}=\overline{U_{2}}\left(1+\varepsilon \mu_{2} \sin \left(\Omega_{2} \mathrm{~T}_{0}\right)\right)
\end{aligned}
$$

Sorting the perturbed equations in orders of $(\varepsilon)$, we have:

U-equation:

$$
\begin{aligned}
& O\left(\varepsilon^{0}\right): D_{0}{ }^{2} \bar{u}_{0}+C 21 D_{0} \bar{u}_{0}{ }^{\prime} \bar{U}_{1}+C 22 D_{0} \bar{u}_{0}{ }^{\prime \prime} \bar{U}_{2}+C 31 \bar{u}_{0}{ }^{\prime \prime} \bar{U}_{1}{ }^{2}+C 32 \bar{u}_{0}{ }^{\prime \prime} \bar{U}_{2}{ }^{2}- \\
& C 5 \bar{u}_{0}^{\prime \prime}=0 \\
& O\left(\varepsilon^{1}\right): D_{0}{ }^{2} \bar{u}_{1}+C 21 D_{0} \bar{u}_{1}^{\prime} \bar{U}_{1}+C 22 D_{0} \bar{u}_{1}^{\prime} \bar{U}_{2}+2 D_{0} D_{1} \bar{u}_{0}+C 31 \bar{u}_{1}^{\prime \prime} \bar{U}_{1}^{2}+ \\
& C 32 \bar{u}_{1}^{\prime \prime} \bar{U}_{2}{ }^{2}+C 21 D_{0} \bar{u}_{1}^{\prime} \bar{U}_{1}+C 22 D_{0} \bar{u}_{1}^{\prime} \bar{U}_{2}-C 5 \bar{u}_{1}^{\prime \prime}-\bar{v}_{0}^{\prime \prime \prime \prime} \bar{v}_{0}{ }^{\prime}-C 7^{\prime}+\gamma- \\
& \bar{v}_{0}^{\prime \prime} \bar{v}_{0}^{\prime \prime \prime}+C 6 \bar{v}_{0}^{\prime} \bar{v}_{0}^{\prime \prime}+\mathrm{C} 21 D_{1} \bar{u}_{0}^{\prime} \bar{U}_{1}+C 22 D_{1} \bar{u}_{0}^{\prime} \bar{U}_{2}+C 11 \Omega_{1} \mu_{1} \cos \left(\Omega_{1} T 0\right) \bar{U}_{1}+ \\
& C 12 \Omega_{2} \mu_{2} \cos \left(\Omega_{2} T 0\right) \bar{U}_{2}+2 C 31 \mu_{1} \sin \left(\Omega_{1} T 0\right) \bar{U}_{1}^{2} \bar{u}_{0}{ }^{\prime \prime}+ \\
& 2 C 32 \mu_{2} \sin \left(\Omega_{2} T 0\right) \bar{U}_{2}{ }^{2} \bar{u}_{0}{ }^{\prime \prime}+C 21 \mu_{1} \sin \left(\Omega_{1} T 0\right) D_{0} \bar{U}_{1} \bar{u}_{0}{ }^{\prime}+ \\
& C 22 \mu_{2} \sin \left(\Omega_{2} T 0\right) D_{0} \bar{U}_{2} \bar{u}_{0}{ }^{\prime}+C 41 \Omega_{1} \mu_{1} \cos \left(\Omega_{1} T 0\right) \bar{U}_{1} \bar{u}_{0}{ }^{\prime}+ \\
& C 42 \Omega_{2} \mu_{2} \cos \left(\Omega_{2} T 0\right) \bar{U}_{2} \bar{u}_{0}{ }^{\prime}=0
\end{aligned}
$$

V-equation:

$$
\begin{aligned}
& O\left(\varepsilon^{0}\right): D_{0}{ }^{2} \bar{v}_{0}-C 7 \bar{v}_{0}{ }^{\prime \prime}+\bar{v}_{0}{ }^{\prime \prime \prime \prime}+C 21 D_{0} \bar{v}_{0}{ }^{\prime} \bar{U}_{1}+C 22 D_{0} \bar{v}_{0}{ }^{\prime} \bar{U}_{2}+C 31 \bar{v}_{0}{ }^{\prime \prime} \bar{U}_{1}{ }^{2}+ \\
& C 32 \bar{v}_{0}{ }^{\prime \prime} \bar{U}_{2}{ }^{2}-a C 31 \bar{v}_{0}{ }^{\prime \prime} \bar{U}_{1}{ }^{2}-a C 32 \bar{v}_{0}{ }^{\prime \prime} \bar{U}_{2}{ }^{2}=0 \\
& O\left(\varepsilon^{1}\right): \quad D_{0}{ }^{2} \bar{v}_{1}-C 7 \bar{v}_{1}{ }^{\prime \prime}+\bar{v}_{1}{ }^{\prime \prime \prime \prime}-\bar{u}_{0}{ }^{\prime \prime \prime \prime} \bar{v}_{0}{ }^{\prime}-2 \bar{u}_{0}{ }^{\prime} \bar{v}_{0}{ }^{\prime \prime \prime \prime}-4 \bar{u}_{0}{ }^{\prime \prime} \bar{v}_{0}{ }^{\prime \prime \prime}- \\
& 3 \bar{v}_{0}{ }^{\prime \prime} \bar{v}_{0}{ }^{\prime \prime \prime}-2 \bar{v}_{0}{ }^{3 \prime \prime}-2 \bar{v}_{0}{ }^{\prime \prime \prime \prime} \bar{v}_{0}{ }^{\prime}+2 D_{0} D_{1} \bar{v}_{0}+C 31 \bar{v}_{1}{ }^{\prime \prime} \bar{U}_{1}{ }^{2}+C 32 \bar{v}_{1}{ }^{\prime \prime} \bar{U}_{2}{ }^{2}- \\
& 8 \bar{v}_{0}{ }^{\prime} \bar{v}_{0}{ }^{\prime \prime} \bar{v}_{0}{ }^{\prime \prime \prime}+C 6 \bar{u}_{0}{ }^{\prime} \bar{v}_{0}{ }^{\prime \prime}+C 6 \bar{u}_{0}{ }^{\prime \prime} \bar{v}_{0}{ }^{\prime}+\frac{3}{2} C 6 \bar{v}_{0}{ }^{\prime} \bar{v}_{0}{ }^{\prime \prime}+C 21 D_{0} \bar{v}_{0}{ }^{\prime} \bar{U}_{1}+ \\
& C 22 D_{0} \bar{v}_{0}{ }^{\prime} \bar{U}_{2}+C 21 D_{1} \bar{v}_{0}^{\prime} \bar{U}_{1}+C 22 D_{1} \bar{v}_{0}{ }^{\prime} \bar{U}_{2}-a C 31 \bar{v}_{1}{ }^{\prime \prime} \bar{U}_{1}{ }^{2}-a C 32 \bar{v}_{1}{ }^{\prime \prime} \bar{U}_{2}{ }^{2}+ \\
& 2 C 31 \mu_{1} \sin \left(\Omega_{1} T 0\right) \bar{U}_{1}^{2} \bar{v}_{0}^{\prime \prime}+2 C 32 \mu_{2} \sin \left(\Omega_{2} T 0\right) \bar{U}_{2}^{2} \bar{v}_{0}^{\prime \prime}+ \\
& C 21 \mu_{1} \sin \left(\Omega_{1} T 0\right) D_{0} \bar{U}_{1} \bar{v}_{0}{ }^{\prime}+C 22 \mu_{2} \sin \left(\Omega_{2} T 0\right) D_{0} \bar{U}_{2} \bar{v}_{0}{ }^{\prime}- \\
& 2 a C 31 \mu_{1} \sin \left(\Omega_{1} T 0\right) \bar{U}_{1}^{2} \bar{v}_{0}{ }^{\prime \prime}-2 a C 32 \mu_{2} \sin \left(\Omega_{2} T 0\right) \bar{U}_{2}{ }^{2} \bar{v}_{0}{ }^{\prime \prime}+ \\
& C 41 \Omega_{1} \mu_{1} \cos \left(\Omega_{1} T 0\right) \bar{U}_{1} \bar{v}_{0}{ }^{\prime}+C 42 \Omega_{2} \mu_{2} \cos \left(\Omega_{2} T 0\right) \bar{U}_{2} \bar{v}_{0}{ }^{\prime}=0
\end{aligned}
$$

The planar natural frequencies and mode shapes can be obtained by solving the leading order equations. The homogeneous solution of the leading order equations (17) and (19) can be expressed as:

$$
\begin{aligned}
& \bar{u}\left(x, T_{0}, T_{1}\right)_{0}=\phi(x)_{n} \exp \left(i \omega_{n} T_{0}\right)+C C \\
& \bar{v}\left(x, T_{0}, T_{1}\right)_{0}=\eta(x)_{n} \exp \left(i \lambda_{n} T_{0}\right)+C C
\end{aligned}
$$

Where $(C C)$ is the complex conjugate, $\phi(x)_{n}$ and $\eta(x)_{n}$ are the complex modal functions for the axial and transverse vibrations for each mode (n) and, $\omega_{n}$ and $\lambda_{n}$ are the eigenvalues for the axial and transverse vibrations for each mode (n). 


\subsection{Principal parametric resonance}

Substituting the homogeneous solution of the leading order equations into the equations (18) and (20) gives;

$$
\begin{aligned}
& D_{0}{ }^{2} \bar{u}_{1}-C 5 \bar{u}_{1}^{\prime \prime}+C 21 D_{0} \bar{u}_{1}^{\prime} \bar{U}_{1}+C 22 D_{0} \bar{u}_{1}^{\prime} \bar{U}_{2}+C 31 \bar{u}_{1}^{\prime \prime} \bar{U}_{1}{ }^{2}+C 32 \bar{u}_{1}^{\prime \prime} \bar{U}_{2}{ }^{2}= \\
& -\left(C 21 \frac{\partial X(T 1)}{\partial T 1} \frac{\partial \phi(x)}{\partial x} \bar{U}_{1}+C 22 \frac{\partial X(T 1)}{\partial T 1} \frac{\partial \phi(x)}{\partial x} \bar{U}_{2}+2 i \frac{\partial X(T 1)}{\partial T 1} \omega\right) \exp \left(i \omega T_{0}\right)+ \\
& Y(T 1)^{2}\left(\frac{\partial \eta(x)}{\partial x} \frac{\partial^{4} \eta(x)}{\partial x^{4}}+\frac{\partial^{2} \eta(x)}{\partial x^{2}} \frac{\partial^{3} \eta(x)}{\partial x^{3}}-C 6 \frac{\partial \eta(x)}{\partial x} \frac{\partial^{2} \eta(x)}{\partial x^{2}}\right) \exp \left(2 i \lambda T_{0}\right)+ \\
& {\left[C 32 \mu_{2} \frac{\partial^{2} \phi(x)}{\partial x^{2}} \exp \left(i \Omega_{2} T_{0}\right) \bar{U}_{2}{ }^{2} i-\frac{1}{2}\left(C 21 \mu_{1} \frac{\partial \phi(x)}{\partial x} \exp \left(-i \Omega_{1} T_{0}\right) \bar{U}_{1} \omega\right)+\right.} \\
& \frac{1}{2}\left(C 21 \mu_{1} \frac{\partial \phi(x)}{\partial x} \exp \left(i \Omega_{1} T_{0}\right) \bar{U}_{1} \omega\right)-\frac{1}{2}\left(C 22 \mu_{2} \frac{\partial \phi(x)}{\partial x} \exp \left(-i \Omega_{2} T_{0}\right) \bar{U}_{2} \omega\right)+ \\
& \frac{1}{2}\left(C 22 \mu_{2} \frac{\partial \phi(x)}{\partial x} \exp \left(i \Omega_{2} T_{0}\right) \bar{U}_{2} \omega\right)-\frac{1}{2}\left(C 41 \Omega_{1} \mu_{1} \frac{\partial \phi(x)}{\partial x} \exp \left(-i \Omega_{1} T_{0}\right) \bar{U}_{1}\right)- \\
& \frac{1}{2}\left(C 41 \Omega_{1} \mu_{1} \frac{\partial \phi(x)}{\partial x} \exp \left(i \Omega_{1} T_{0}\right) \bar{U}_{1}\right)-\frac{1}{2}\left(C 42 \Omega_{2} \mu_{2} \frac{\partial \phi(x)}{\partial x} \exp \left(-i \Omega_{2} T_{0}\right) \bar{U}_{2}\right)- \\
& \frac{1}{2}\left(C 42 \Omega_{2} \mu_{2} \frac{\partial \phi(x)}{\partial x} \exp \left(i \Omega_{2} T_{0}\right) \bar{U}_{2}\right)-C 32 \mu_{2} \frac{\partial^{2} \phi(x)}{\partial x^{2}} \exp \left(-i \Omega_{2} T_{0}\right) \bar{U}_{2}{ }^{2} i- \\
& \left.C 31 \mu_{1} \frac{\partial^{2} \phi(x)}{\partial x^{2}} \exp \left(-i \Omega_{1} T_{0}\right) \bar{U}_{1}{ }^{2} i+C 31 \mu_{1} \frac{\partial^{2} \phi(x)}{\partial x^{2}} \exp \left(i \Omega_{1} T_{0}\right) \bar{U}_{1}{ }^{2} i\right] X(T 1) \exp \left(i \omega T_{0}\right)+ \\
& {\left[C 32 \mu_{2} \frac{\partial^{2} \overline{\phi(x)}}{\partial x^{2}} \exp \left(i \Omega_{2} T_{0}\right) \bar{U}_{2}{ }^{2} i+\frac{1}{2}\left(C 21 \mu_{1} \frac{\partial \overline{\phi(x)}}{\partial x} \exp \left(i \Omega_{1} T_{0}\right) \bar{U}_{1} \omega\right)+\right.} \\
& \frac{1}{2}\left(C 22 \mu_{2} \frac{\partial \overline{\phi(x)}}{\partial x} \exp \left(i \Omega_{2} T_{0}\right) \bar{U}_{2} \omega\right)-\frac{1}{2}\left(C 41 \Omega_{1} \mu_{1} \frac{\partial \overline{\phi(x)}}{\partial x} \exp \left(i \Omega_{1} T_{0}\right) \bar{U}_{1}\right)- \\
& \frac{1}{2}\left(C 42 \Omega_{2} \mu_{2} \frac{\partial \overline{\phi(x)}}{\partial x} \exp \left(i \Omega_{2} T_{0}\right) \bar{U}_{2}\right)+ \\
& \left.C 31 \mu_{1} \frac{\partial^{2} \overline{\phi(x)}}{\partial x^{2}} \exp \left(i \Omega_{1} T_{0}\right) \bar{U}_{1}{ }^{2} i\right] \overline{X(T 1)} \exp \left(-i \omega T_{0}\right)+N S T+C C=0 \\
& D_{0}{ }^{2} \bar{v}_{1}-C 7 \bar{v}_{1}^{\prime \prime}+\bar{v}_{1}^{\prime \prime \prime \prime}+C 21 D_{0} \bar{v}_{1}^{\prime} \bar{U}_{1}+C 22 D_{0} \bar{v}_{1}^{\prime} \bar{U}_{2}+C 31 \bar{v}_{1}^{\prime \prime} \bar{U}_{1}^{2}+ \\
& C 32 \bar{v}_{1}^{\prime \prime} \bar{U}_{2}{ }^{2}-a C 31 \bar{v}_{1}^{\prime \prime} \bar{U}_{1}{ }^{2}-a C 32 \bar{v}_{1}{ }^{\prime \prime} \bar{U}_{2}{ }^{2}=\left(-\frac{\partial Y(T 1)}{\partial T 1}\left(C 21 \frac{\partial \eta(x)}{\partial x} \bar{U}_{1}+\right.\right. \\
& \left.C 22 \frac{\partial \eta(x)}{\partial x} \bar{U}_{2}+2 \eta(x) \lambda i\right)+6 Y(T 1)^{2} \overline{Y(T 1)}\left(\frac{\partial \eta(x)}{\partial x}\right)^{2} \frac{\partial \overline{\eta(x)}}{\partial x}+ \\
& 2 Y(T 1)^{2} \overline{Y(T 1)}\left(\frac{\partial \eta(x)}{\partial x}\right)^{2} \frac{\partial^{4} \overline{\eta(x)}}{\partial x^{4}}+4 Y(T 1)^{2} \overline{Y(T 1)} \frac{\partial \eta(x)}{\partial x} \frac{\partial \overline{\eta(x)}}{\partial x} \frac{\partial^{4} \eta(x)}{\partial x^{4}}+ \\
& 8 Y(T 1)^{2} \overline{Y(T 1)} \frac{\partial \eta(x)}{\partial x} \frac{\partial^{2} \overline{\eta(x)}}{\partial x^{2}} \frac{\partial^{3} \eta(x)}{\partial x^{3}}+8 Y(T 1)^{2} \overline{Y(T 1)} \frac{\partial \overline{\eta(x)}}{\partial x} \frac{\partial^{2} \eta(x)}{\partial x^{2}} \frac{\partial^{3} \eta(x)}{\partial x^{3}}- \\
& \text { 3C6. } Y(T 1)^{2} \overline{Y(T 1)} \frac{\partial \eta(x)}{\partial x} \frac{\partial \overline{\eta(x)}}{\partial x} \frac{\partial^{2} \eta(x)}{\partial x^{2}}+8 Y(T 1)^{2} \overline{Y(T 1)} \frac{\partial \eta(x)}{\partial x} \frac{\partial^{2} \eta(x)}{\partial x^{2}} \frac{\partial^{3} \overline{\eta(x)}}{\partial x^{3}}- \\
& \left.\frac{3}{2} C 6 . Y(T 1)^{2} \overline{Y(T 1)}\left(\frac{\partial \eta(x)}{\partial x}\right)^{2} \frac{\partial^{2} \eta(x)}{\partial x^{2}}\right) \exp \left(i \lambda T_{0}\right)+\left(2 X(T 1) \overline{Y(T 1)} \frac{\partial \Phi(x)}{\partial x} \frac{\partial^{4} \overline{\eta(x)}}{\partial x^{4}}+\right. \\
& 4 X(T 1) \overline{Y(T 1)} \frac{\partial^{2} \Phi(x)}{\partial x^{2}} \frac{\partial^{3} \overline{\eta(x)}}{\partial 3}+ \\
& \left.3 X(T 1) \overline{Y(T 1)} \frac{\partial^{2} \overline{\eta(x)}}{\partial 2} \frac{\partial^{3} \Phi(x)}{\partial 3}\right) \exp \left(i \omega T_{0}\right) \exp \left(-i \lambda T_{0}\right)- \\
& \left(C 6 X(T 1) \overline{Y(T 1)} \frac{\partial \Phi(x)}{\partial x} \frac{\partial^{2} \overline{\eta(x)}}{\partial x^{2}}+\right. \\
& \left.C 6 X(T 1) \overline{Y(T 1)} \frac{\partial \overline{\eta(x)}}{\partial x} \frac{\partial^{2} \Phi(x)}{\partial x^{2}}\right) \exp \left(i \omega T_{0}\right) \exp \left(-i \lambda T_{0}\right)+\left[\left(\frac{1}{2}\left(C 22 \mu_{2} \frac{\partial \eta(x)}{\partial x} \bar{U}_{2} \lambda\right)-\right.\right. \\
& \frac{1}{2}\left(C 42 \Omega_{2} \mu_{2} \frac{\partial \eta(x)}{\partial x} \bar{U}_{2}\right)+a C 32 \mu_{2} \frac{\partial^{2} \eta(x)}{\partial x^{2}} \bar{U}_{2}{ }^{2} i- \\
& \left.C 32 \mu_{2} \frac{\partial^{2} \eta(x)}{\partial x^{2}} \bar{U}_{2}^{2} i\right) \exp \left(-i \Omega_{2} T_{0}\right)+\left(\frac{1}{2}\left(C 21 \mu_{1} \frac{\partial \eta(x)}{\partial x} \bar{U}_{1} \lambda\right)-\right. \\
& \frac{1}{2}\left(C 41 \Omega_{1} \mu_{1} \frac{\partial \eta(x)}{\partial x} \bar{U}_{1}\right)+a C 31 \mu_{1} \frac{\partial^{2} \eta(x)}{\partial x^{2}} \bar{U}_{1}{ }^{2} i- \\
& \left.C 31 \mu_{1} \frac{\partial^{2} \eta(x)}{\partial x^{2}} \bar{U}_{1}^{2} i\right) \exp \left(-i \Omega_{1} T_{0}\right)-\left(\frac{1}{2}\left(C 21 \mu_{1} \frac{\partial \eta(x)}{\partial x} \bar{U}_{1} \lambda\right)-\right. \\
& \left.\frac{1}{2}\left(C 41 \Omega_{1} \mu_{1} \frac{\partial \eta(x)}{\partial x} \bar{U}_{1}\right)+a C 31 \mu_{1} \frac{\partial^{2} \eta(x)}{\partial x^{2}} \bar{U}_{1}{ }^{2} i-C 31 \mu_{1} \frac{\partial^{2} \eta(x)}{\partial x^{2}} \bar{U}_{1}{ }^{2} i\right) \exp \left(i \Omega_{1} T_{0}\right)- \\
& \left(\frac{1}{2}\left(C 22 \mu_{2} \frac{\partial \eta(x)}{\partial x} \bar{U}_{2} \lambda\right)-\frac{1}{2}\left(C 42 \Omega_{2} \mu_{2} \frac{\partial \eta(x)}{\partial x} \bar{U}_{2}\right)+a C 32 \mu_{2} \frac{\partial^{2} \eta(x)}{\partial x^{2}} \bar{U}_{2}{ }^{2} i-\right.
\end{aligned}
$$




$$
\begin{aligned}
& \left.\left.C 32 \mu_{2} \frac{\partial^{2} \eta(x)}{\partial x^{2}} \bar{U}_{2}^{2} i\right) \exp \left(i \Omega_{2} T_{0}\right)\right] Y(T 1) \exp \left(i \lambda T_{0}\right)+\left[\left(\frac{1}{2}\left(C 21 \mu_{1} \frac{\partial \overline{\eta(x)}}{\partial x} \bar{U}_{1} \lambda\right)-\right.\right. \\
& \left.\frac{1}{2}\left(C 41 \Omega_{1} \mu_{1} \frac{\partial \overline{\eta(x)}}{\partial x} \bar{U}_{1}\right)-a C 31 \mu_{1} \frac{\partial^{2} \overline{\eta(x)}}{\partial x^{2}} \bar{U}_{1}^{2} i+C 31 \mu_{1} \frac{\partial^{2} \overline{\eta(x)}}{\partial x^{2}} \bar{U}_{1}^{2} i\right) \exp \left(i \Omega_{1} T_{0}\right)+ \\
& \left(\frac{1}{2}\left(C 22 \mu_{2} \frac{\partial \overline{\eta(x)}}{\partial x} \bar{U}_{2} \lambda\right)-\frac{1}{2}\left(C 42 \Omega_{2} \mu_{2} \frac{\partial \overline{\eta(x)}}{\partial x} \bar{U}_{2}\right)-a C 32 \mu_{2} \frac{\partial^{2} \overline{\eta(x)}}{\partial x^{2}} \bar{U}_{2}{ }^{2} i+\right. \\
& \left.\left.C 32 \mu_{2} \frac{\partial^{2} \overline{\eta(x)}}{\partial x^{2}} \bar{U}_{2}^{2} i\right) \exp \left(i \Omega_{2} T_{0}\right)\right] \overline{Y(T 1)} \exp \left(-i \lambda T_{0}\right)+N S T+C C=0
\end{aligned}
$$

Here NST denotes non-secular terms. Examining the equations for expressions that will lead to secular terms, it can be observed that various scenarios exist. However, unlike pulsating single-phase flow, three fundamental parametric resonance problems that are solely associated with the planar dynamics of pipes with pulsating two-phase flow are identified:

- Parametric resonance case with $\Omega 1$ and $\Omega 2$ close to $2 \lambda$ but far from $2 \omega$

- Parametric resonance case with $\Omega 1$ and $\Omega 2$ close to $2 \omega$ but far from $2 \lambda$

- Parametric resonance case with $\Omega 1$ close to $2 \omega$ and $\Omega 2$ close to $2 \lambda$

$\omega$ and $\lambda$ are the axial and transverse natural frequencies. As reported in [4], there is 1:2 internal resonance relationships between the axial and transverse frequencies. However, this study only considers the transverse vibrations with parametric resonance case where $\Omega 1$ and $\Omega 2$ are equal and close to $2 \lambda$ but far from $2 \omega$ for both the internal resonance condition and $(\omega=2 \lambda)$ away from the internal resonance condition $(\omega \neq 2 \lambda)$.

The proximity of nearness is expressed as: $\Omega 1=2 \lambda+\varepsilon \sigma_{2}$ and $\Omega 2=2 \lambda+\varepsilon \sigma_{2}$, (Where $\sigma_{2}$ is the detuning parameter between the forcing frequency and transverse natural frequency).

\subsubsection{When $\boldsymbol{\omega}$ is far from $\mathbf{2} \lambda$ (Away from Internal resonance condition)}

The inner product defined for complex functions on $\{0,1\}$ as: $\langle f, g\rangle=\int_{0}^{1} f \bar{g} d x$.

Solvability criterion demands that the coefficient of $\exp \left(i \omega T_{0}\right)$ and $\exp \left(i \lambda T_{0}\right)$ should vanish [5]. This implies that, $\mathrm{X}\left(\mathrm{T}_{1}\right)$ and $\mathrm{Y}\left(\mathrm{T}_{1}\right)$ should satisfy the following relation:

$$
\begin{aligned}
& \frac{\partial X\left(T_{1}\right)}{\partial T_{1}}=0 \\
& \frac{\partial Y\left(T_{1}\right)}{\partial T_{1}}+N Y\left(T_{1}\right)^{2} \overline{Y\left(T_{1}\right)}+M \overline{Y\left(T_{1}\right)} \exp \left(i \sigma_{2} T_{1}\right)=0
\end{aligned}
$$

Where: $N=\frac{\int_{0}^{1}[\mathrm{~B} 2] \overline{\eta(x)} d x}{-\int_{0}^{1}[\mathrm{~B} 1] \overline{\eta(x)} d x}, M=\frac{\int_{0}^{1}[\mathrm{~B} 4+\mathrm{B} 5] \overline{\eta(x)} d x}{-\int_{0}^{1}[\mathrm{~B} 1] \overline{\eta(x)} d x}$.

B1, B2, B4 and B5 are defined in the appendix.

Expressing the solutions of equations (18) and (19) in polar form as:

$$
\begin{aligned}
& Y\left(T_{1}\right)=\frac{1}{2} \alpha y\left(T_{1}\right) e^{i \beta y\left(T_{1}\right)} \text { and } \overline{Y\left(T_{1}\right)}=\frac{1}{2} \alpha y\left(T_{1}\right) e^{-i \beta y\left(T_{1}\right)} \\
& X\left(T_{1}\right)=\frac{1}{2} \alpha x\left(T_{1}\right) e^{i \beta x\left(T_{1}\right)} \text { and } \overline{X\left(T_{1}\right)}=\frac{1}{2} \alpha x\left(T_{1}\right) e^{-i \beta x\left(T_{1}\right)}
\end{aligned}
$$

Solving equation (25) for $\mathrm{X}\left(T_{1}\right)$, gives a constant amplitude solution up to the 1 st order approximation: 


$$
X\left(T_{1}\right)=\alpha x_{0}
$$

Adopting the assumed solutions in equation (28), the modulation equations are formed. With ' $R$ ' the real parts and ' $I$ ' the imaginary parts of $M$ and $N$. The stationary solutions are obtained as:

$$
\begin{aligned}
& \psi=\tan ^{-1}\left(\frac{N I \alpha y\left(T_{1}\right)^{2}+2 \sigma_{2}}{N R \alpha y\left(T_{1}\right)^{2}}\right)-\tan ^{-1}\left(\frac{M I}{M R}\right) \\
& \left(N I^{2}+N R^{2}\right) \alpha y\left(T_{1}\right)^{4}+4 N I \sigma_{2} \alpha y\left(T_{1}\right)^{2}-16 M R^{2}-16 M I^{2}+4 \sigma_{2}^{2}=0
\end{aligned}
$$

With $\mathrm{A}=N I^{2}+N R^{2}, \mathrm{~B}=4 N I \sigma_{2}, \mathrm{C}=4{\sigma_{2}}^{2}-16 M R^{2}-16 M I^{2}$

The solution of equation (24) will produce four roots of $\alpha y\left(T_{1}\right)$ :

$$
\alpha y\left(T_{1}\right)= \pm \frac{\sqrt{-\frac{2\left(\mathrm{~B}+\sqrt{\mathrm{B}^{2}-4 \mathrm{AC}}\right)}{\mathrm{A}}}}{2} \text { or } \pm \frac{\sqrt{-\frac{2\left(\mathrm{~B}-\sqrt{\mathrm{B}^{2}-4 \mathrm{AC}}\right)}{\mathrm{A}}}}{2}
$$

However, the acceptable solution of $\alpha y\left(T_{1}\right)$ is the root of the quartic equation (31) that is real and positive. Considering: $T_{0}=t, \alpha y\left(T_{1}\right)_{n}=\alpha y_{n}, \beta y\left(T_{1}\right)_{n}=\frac{\sigma_{2 n} T_{1}-\psi_{n}}{2}, \Omega 1=\Omega 2=\Omega$.

With the stability condition fulfilled, the particular solution of equation (24) away from internal resonance condition is obtained as:

$$
v_{1}=\frac{G 1 \alpha y\left(T_{1}\right)^{3}}{4} \cos \left(3\left(\beta y\left(T_{1}\right)+T_{0} \lambda\right)\right)+G 2 \alpha y\left(T_{1}\right) \cos \left(\beta y\left(T_{1}\right)+T_{0}(\Omega+\lambda)\right)
$$

The first order approximate solution of $\bar{v}(x, t)$ without internal resonance is expressed as:

$$
\bar{v}(x, t)=\sum_{\mathrm{n}=1}^{\infty} \alpha y_{n}\left|\eta(x)_{n}\right| \cos \left(\frac{(t \Omega-\psi)}{2}+\varphi y_{n}\right)+O(\varepsilon)
$$

The phase angles $\left(\varphi y_{n}\right)$ are given by: $\tan \left(\varphi y_{n}\right)=\frac{\operatorname{Im}\left\{\eta(x)_{n}\right\}}{\operatorname{Re}\left\{\eta(x)_{n}\right\}}$.

The stability of the solutions is determined by examining the eigenvalues of the Jacobian of the modulation equations. The Jacobian is expressed as:

$$
J=\left[\begin{array}{cc}
-\frac{N R \alpha y\left(T_{1}\right)}{2} & M I \cos (\psi)+M R \sin (\psi) \\
-\frac{N R \alpha y\left(T_{1}\right)}{2} M I \sin (\psi) & M I \sin (\psi)-M R \cos (\psi)
\end{array}\right]
$$

The characteristics equation is obtained by solving for the eigenvalues of the Jacobian,

$$
|J-\vartheta I|=0 \text {, where } \vartheta \text { are the eigenvalues. }
$$

However, to assess the stability of the nonlinear solution (nontrivial solution), we substitute the solutions of $\alpha y\left(T_{1}\right)$ as obtained from equation (32) into the characteristic equation:

$$
\vartheta^{2}+C 1 \vartheta+C 2=0
$$




$$
\begin{aligned}
& C 1=M R \cos (\psi)-M I \sin (\psi)+\frac{N R \alpha y\left(T_{1}\right)}{2}, \\
& C 2=\frac{M I N I \alpha y\left(T_{1}\right) \cos (\psi)}{2}+\frac{M R N R \alpha y\left(T_{1}\right) \cos (\psi)}{2}-\frac{M I N R \alpha y\left(T_{1}\right) \sin (\psi)}{2}+\frac{M R N I \alpha y\left(T_{1}\right) \sin (\psi)}{2},
\end{aligned}
$$

Adopting the Routh-Hurwitz criteria, the nonlinear fixed point solution of $\alpha y\left(T_{1}\right)$ is stable only if:

$$
C 1>0 \text { and } C 2>0 \text {. }
$$

\subsubsection{When $\boldsymbol{\omega}$ is close to $\mathbf{2} \lambda$ (Internal resonance condition)}

When $\omega=2 \lambda$, another detuning parameter $\sigma_{1}$, is introduced: $\omega=2 \lambda+\varepsilon \sigma_{1}$, therefore; $2 \lambda T_{0}=\omega T_{0}-\sigma_{1} T_{1}$ and $(\omega-\lambda) T_{0}=\lambda T_{0}+\sigma_{1} T_{1}$, with $\varepsilon T_{0}=T_{1}$.

In this case, solvability condition demands that, $\mathrm{X}\left(\mathrm{T}_{1}\right)$ and $\mathrm{Y}\left(\mathrm{T}_{1}\right)$ satisfy the following relation:

$$
\begin{aligned}
& \frac{\partial X\left(T_{1}\right)}{\partial T_{1}}-J 2 Y\left(T_{1}\right)^{2} \exp \left(-\sigma_{1} T_{1} i\right)=0 \\
& \frac{\partial Y\left(T_{1}\right)}{\partial T_{1}}+K 2 Y\left(T_{1}\right)^{2} \overline{Y\left(T_{1}\right)}+K 3 \overline{Y\left(T_{1}\right)} \exp \left(i \sigma_{2} T_{1}\right)+K 4 \overline{Y\left(T_{1}\right)} \exp \left(i \sigma_{2} T_{1}\right)=0
\end{aligned}
$$

Where: $J 2=\frac{\int_{0}^{1}[A 2] \overline{\phi(x)} d x}{-\int_{0}^{1}[A 1] \overline{\phi(x)} d x}, K 2=\frac{\int_{0}^{1}[\mathrm{~B} 2] \overline{\eta(x)} d x}{-\int_{0}^{1}[\mathrm{~B} 1] \overline{\eta(x)} d x}, K 3=\frac{\int_{0}^{1}[\mathrm{~B} 3] \overline{\eta(x)} d x}{-\int_{0}^{1}[\mathrm{~B} 1] \overline{\eta(x)} d x}, K 4=\frac{\int_{0}^{1}[\mathrm{~B} 4+\mathrm{B} 5] \overline{\eta(x)} d x}{-\int_{0}^{1}[\mathrm{~B} 1] \overline{\eta(x)} d x}$.

A1, A2, B1, B2, B3, B4 and B5 are defined in the appendix.

To derive the corresponding modulation equations, the assumed solutions in equations (27) and (28) are adopted. From the stationary solutions of the modulation equations, the linear solutions are obtained as; $\alpha x\left(T_{1}\right)=\alpha y\left(T_{1}\right)=0$. Representing ' $\mathrm{R}$ ' as the real parts and ' $\mathrm{I}$ ' as the imaginary parts of $\mathrm{J} 2, \mathrm{~K} 2, \mathrm{~K} 3$ and $\mathrm{K} 4$, the stationary solutions are obtained for the coupled system as:

$$
\begin{gathered}
\psi 1=-\tan ^{-1}\left(\frac{J 2 R}{J 2 I}\right) \\
\begin{array}{c}
\psi=\tan ^{-1}\left(\frac{K 2 \operatorname{I\alpha y}\left(T_{1}\right)^{2}+2 \sigma_{2}+2 K 3 \operatorname{I\alpha x}\left(T_{1}\right) \cos (\psi 1)+2 K 3 \operatorname{Rox}\left(T_{1}\right) \sin (\psi 1)}{K 2 R \alpha y\left(T_{1}\right)^{2}+2 K 3 \operatorname{R\alpha } x\left(T_{1}\right) \cos (\psi 1)+2 K 3 \operatorname{I\alpha x}\left(T_{1}\right) \sin (\psi 1)}\right) \\
-\tan ^{-1}\left(\frac{K 5 I}{K 5 R}\right)
\end{array}
\end{gathered}
$$

Where $C S=\cos (\psi 1), S S=\sin (\psi 1)$, and other notations in appendix, a quartic equation is obtained in terms of $\alpha y\left(T_{1}\right)$ as:

$$
\text { A. } \alpha y\left(T_{1}\right)^{4}+B \cdot \alpha y\left(T_{1}\right)^{2}+C=0
$$

Where:

$A=J 2 I^{2} K 3 I^{2}+J 2 I^{2} K 3 R^{2}+J 2 R^{2} K 3 I^{2}+J 2 R^{2} K 3 R^{2}+K 2 I^{2} \sigma_{1}{ }^{2}+K 2 I^{2}{\sigma_{2}}^{2}+$ $K 2 R^{2} \sigma_{1}{ }^{2}+K 2 R^{2} \sigma_{2}{ }^{2}-2 K 2 I^{2} \sigma_{1} \sigma_{2}-2 K 2 R^{2} \sigma_{1} \sigma_{2}+2 K 2 I K 3 I \sigma_{1} C S \sqrt{J 2 I^{2}+J 2 R^{2}}-$ $2 K 2 I K 3 I \sigma_{2} C S \sqrt{J 2 I^{2}+J 2 R^{2}}+2 K 2 R K 3 R \sigma_{1} C S \sqrt{J 2 I^{2}+J 2 R^{2}}-$ 
$2 K 2 R K 3 R \sigma_{2} C S \sqrt{J 2 I^{2}+J 2 R^{2}}+2 K 2 I K 3 R \sigma_{1} S S \sqrt{J 2 I^{2}+J 2 R^{2}}-$

$2 K 3 I K 2 R \sigma_{1} S S \sqrt{J 2 I^{2}+J 2 R^{2}}-2 K 2 I K 3 R \sigma_{2} S S \sqrt{J 2 I^{2}+J 2 R^{2}}+$ $2 K 3 I K 2 R \sigma_{2} S S \sqrt{J 2 I^{2}+J 2 R^{2}}$.

$B=4 K 2 I \sigma_{2}{ }^{3}-8 K 2 I \sigma_{1}{\sigma_{2}}^{2}-4 K 2 I \sigma_{1}{ }^{2} \sigma_{2}-4 K 3 I \sigma_{2}{ }^{2} C S \sqrt{J 2 I^{2}+J 2 R^{2}}-$

$4 K 3 R \sigma_{2}{ }^{2} S S \sqrt{J 2 I^{2}+J 2 R^{2}}+4 K 3 I \sigma_{1} \sigma_{2} C S \sqrt{J 2 I^{2}+J 2 R^{2}}+4 K 3 R \sigma_{1} \sigma_{2} S S \sqrt{J 2 I^{2}+J 2 R^{2}}$,

$C=4{\sigma_{2}}^{4}-8 \sigma_{1}{\sigma_{2}}^{3}-16 K 4 I^{2}{\sigma_{1}}^{2}-16 K 4 I^{2}{\sigma_{1}}^{2}-16 K 4 R^{2}{\sigma_{2}}^{2}+4{\sigma_{1}}^{2}{\sigma_{2}}^{2}+32 K 4 I^{2} \sigma_{1} \sigma_{2}+$ $32 K 4 R^{2} \sigma_{1} \sigma_{2}$.

The solution of equation (41) will produce four roots of $\alpha y\left(T_{1}\right)$ :

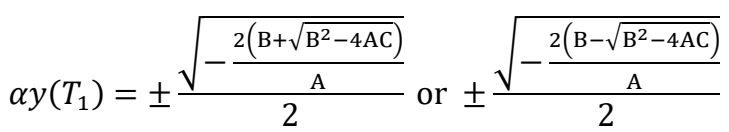

However, the acceptable solution of $\alpha y\left(T_{1}\right)$ is the root of the quartic equation (41) that is real and positive. The expression for estimating $\alpha x\left(T_{1}\right)$ is obtained as a function of $\alpha y\left(T_{1}\right)$ as:

$$
\alpha x\left(T_{1}\right)=\sqrt{\frac{\alpha y\left(T_{1}\right)^{4}\left(J 2 I^{2}+J 2 R^{2}\right)}{4\left(\sigma_{2}-\sigma_{1}\right)}}
$$

Considering: $\mathrm{T}_{0}=t, \alpha x\left(\mathrm{~T}_{1}\right)_{n}=\alpha x_{n}, \alpha y\left(\mathrm{~T}_{1}\right)_{n}=\alpha y_{n}$, and $\Omega 1=\Omega 2=\Omega$.

With the solvability condition fulfilled, the particular solution of equation (17) is obtained as:

$$
\begin{gathered}
v_{1}=\frac{G 1 \alpha y\left(T_{1}\right)^{3}}{4} \cos \left(3\left(\beta y\left(T_{1}\right)+T_{0} \lambda\right)\right)+G 2 \alpha y\left(T_{1}\right) \cos \left(\beta y\left(T_{1}\right)+T_{0}(\Omega+\lambda)\right) \\
+\frac{G 3 \alpha x\left(T_{1}\right) \alpha y\left(T_{1}\right)}{2} \cos \left(\beta x\left(T_{1}\right)+\beta y\left(T_{1}\right)+T_{0}(\omega+\lambda)\right)
\end{gathered}
$$

The first order approximate solution of $\bar{v}(x, t)$ with internal resonance is expressed as:

$$
\bar{v}(x, t)=\sum_{\mathrm{n}=1}^{\infty} \alpha y_{n}\left|\eta(x)_{n}\right| \cos \left(\frac{t \Omega}{2}-\frac{\psi 2_{n}}{2}+\varphi y_{n}\right)+O(\varepsilon)
$$

The phase angles $\varphi x_{n}$ and $\varphi y_{n}$ are given by:

$\tan \left(\varphi x_{n}\right)=\frac{\operatorname{Im}\left\{\phi(x)_{n}\right\}}{\operatorname{Re}\left\{\phi(x)_{n}\right\}}, \tan \left(\varphi y_{n}\right)=\frac{\operatorname{Im}\left\{\eta(x)_{n}\right\}}{\operatorname{Re}\left\{\eta(x)_{n}\right\}}$.

The stability of the solutions is determined by examining the eigenvalues of the Jacobian of the modulation equations. The Jacobian is expressed as:

$$
J=\left[\begin{array}{ccccc}
\frac{K 3 P 2}{2}-\frac{K 2 Q 1^{2}}{4}-K 4-\frac{3 K 2 P 1^{2}}{4} & \frac{K 3 Q 2}{2}-\frac{\sigma_{2}}{2}-\frac{K 2 P 1 Q 1}{2} & \frac{K 3 P 1}{2} & \frac{K 3 Q 1}{2} \\
\frac{\sigma_{2}}{2}+\frac{K 3 Q 2}{2}-\frac{K 2 P 1 Q 1}{2} & K 4-\frac{3 K 2 Q 1^{2}}{4}-\frac{K 2 P 1^{2}}{4}-\frac{K 3 P 2}{2} & -\frac{K 3 Q 1}{2} & \frac{K 3 P 1}{2} \\
-J 2 P 1 & J 2 Q 1 & 0 & \sigma_{1}-\sigma_{2} \\
-J 2 Q 1 & -J 2 P 1 & \sigma_{2}-\sigma_{1} & 0
\end{array}\right]
$$


The characteristics equation is obtained by solving for the eigenvalues of the Jacobian, $|J-\vartheta I|=0$, where $\vartheta$ are the eigenvalues.

To assess the stability of the linear solution (trivial solution), we substitute $\mathrm{Pn}=\mathrm{Qn}=0$ in the characteristic equation:

$$
\begin{aligned}
& \vartheta^{4}+\left(\sigma_{1}{ }^{2}-K 4^{2}+2 \sigma_{1} \sigma_{2}+\frac{5 \sigma_{2}{ }^{2}}{14}\right) \vartheta^{2}+2 K 4^{2} \sigma_{1} \sigma_{2}-K 4^{2} \sigma_{1}-K 4^{2} \sigma_{2}+\frac{\left(\sigma_{1} \sigma_{2}\right)^{2}}{4}- \\
& \frac{\sigma_{1} \sigma_{2}{ }^{3}}{2}+\frac{\sigma_{2}{ }^{4}}{4}=0
\end{aligned}
$$

The trivial solution of $\alpha x\left(\mathrm{~T}_{1}\right)$ and $\alpha y\left(\mathrm{~T}_{1}\right)$, is unstable if at least one eigenvalue has a positive real part, otherwise it is stable. However, to analyze the stability of the nonlinear solution (nontrivial solution), we substitute the solutions of ay and ax as obtained from equations (42) and (43) into the characteristic equation:

$$
\begin{aligned}
& \vartheta^{4}+C 1 \vartheta^{3}+C 2 \vartheta^{2}+C 3 \vartheta+C 4=0 \\
& C 1=K 2 \alpha y\left(\mathrm{~T}_{1}\right)^{2}, \\
& C 2=\frac{K 2^{2} \alpha y\left(\mathrm{~T}_{1}\right)^{4}}{4}+\sigma_{1}{ }^{2}-2 \sigma_{1} \sigma_{2}+\sigma_{2}{ }^{2}-J 2 K 3 \alpha y\left(\mathrm{~T}_{1}\right)^{2}, \\
& C 3=\frac{K 2 \alpha y\left(\mathrm{~T}_{1}\right)\left(2 \sigma_{1}{ }^{2}-2 J 2 K 3 \alpha y\left(\mathrm{~T}_{1}\right)^{2}-4 \sigma_{1} \sigma_{2}+2 \sigma_{2}{ }^{2}\right)}{2}, \\
& C 4=\frac{J 2^{2} J 3 K 2 \alpha y\left(\mathrm{~T}_{1}\right)^{4}}{4}-\frac{J 2 K 3 \alpha y\left(\mathrm{~T}_{1}\right)^{2} \sigma_{1} \sigma_{2}}{2}+\frac{J 2 K 3 \alpha y\left(\mathrm{~T}_{1}\right)^{2} \sigma_{2}{ }^{2}}{2}+\frac{K 2^{2} \alpha y\left(\mathrm{~T}_{1}\right)^{4} \sigma_{1}{ }^{2}}{4}-\frac{K 2^{2} \alpha y\left(\mathrm{~T}_{1}\right)^{4} \sigma_{1} \sigma_{2}}{2}+\frac{K 2^{2} \alpha y\left(\mathrm{~T}_{1}\right)^{4} \sigma_{2}{ }^{2}}{4} .
\end{aligned}
$$

Adopting the Routh-Hurwitz criteria, the nonlinear fixed point solution of ax and ay is stable only if:

$$
C 1>0, C 3>0, C 4>0 \text { and } C 1 C 2 C 3>\left(C 3^{2}+C 1^{2} C 4\right)
$$

\section{Results and Discussion}

The numerical simulation considered a cantilever pipe with external diameter of $11.38 \mathrm{~mm}$, internal diameter of $9.25 \mathrm{~mm}$ and length of $146.7 \mathrm{~mm}$. Its density is $7800 \mathrm{~kg} / \mathrm{m}^{3}$. The pipe conveys air/water two-phase flow with void fractions of $0.1,0.3$, and 0.5 . Critical mixture flow velocities of 11.5, 12.5 and 14.6 for the respective void fractions are obtained from Argand diagram. Pre-critical velocity of 2 , and a post-critical velocity of 16 are simulated to assess the pre-buckling and post-buckling behaviour of the pipe as it responds to parametric forced vibrations of the pulsating flow.

\subsection{Results for $\omega$ far from $2 \lambda$ (Away from internal resonance condition)}

In the absence of internal resonance, the near resonant frequency response of the system is identical to a Duffing system with parametric excitation or the nonlinear Mathieu's equation. Figure 2 shows that in all cases, the peak of the response tilts to the left; this indicates the presence of nonlinear restoring forces of the softening type in the dynamics. In addition, at post-critical flow condition, higher void fractions of 0.3 and 0.5 resulted to a transition between subcritical and supercritical pitchfork bifurcation compared with lower void fraction and the pre-critical flow condition results. The transverse response when the pulsation frequency is perfectly tuned to the transverse natural frequency for all the void fractions as depicted in Figure 5 of Appendix 1 are observed to be with beating time series and dense phase plane orbits which fills up the phase plane. The spectra density 
plots exhibited two concentrated peak which is confirms a quasiperiodic motion with two dominating frequencies.

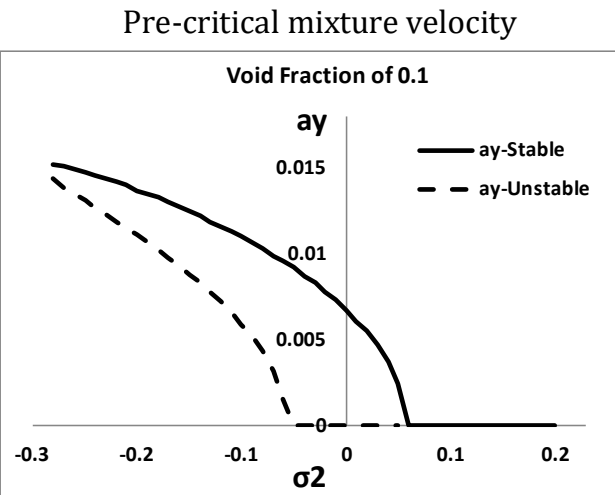

(a)

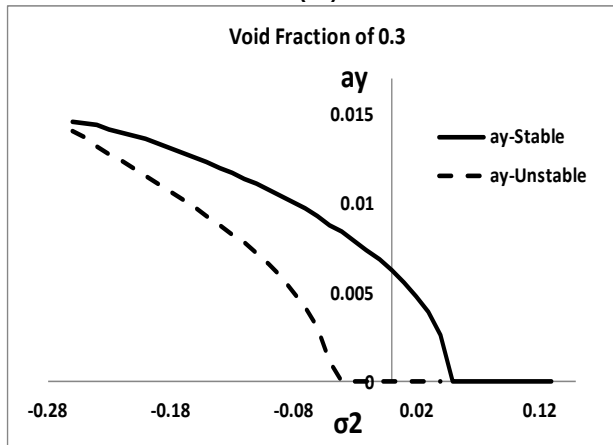

(c)

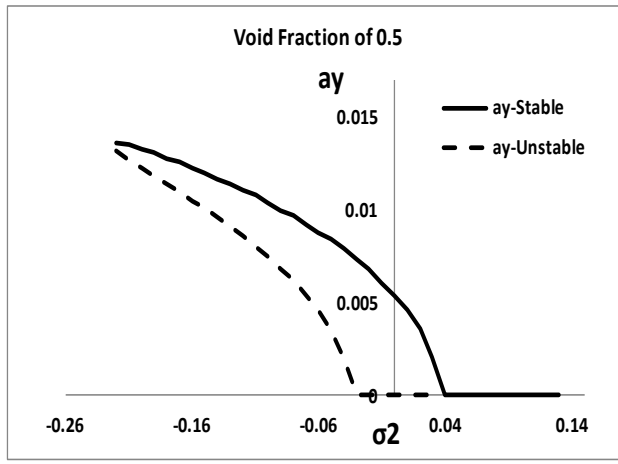

(e)
Post-critical mixture velocity

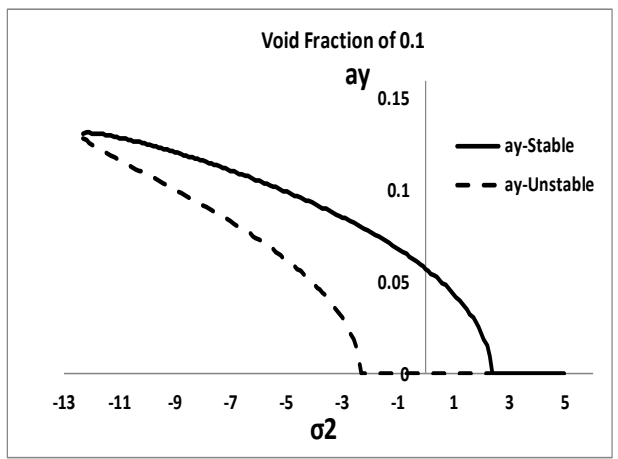

(b)

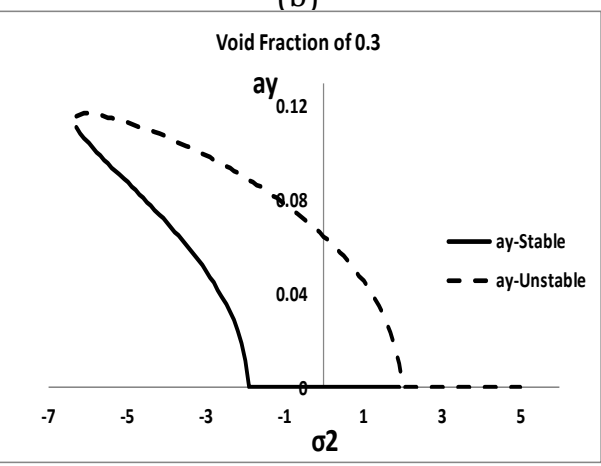

(d)

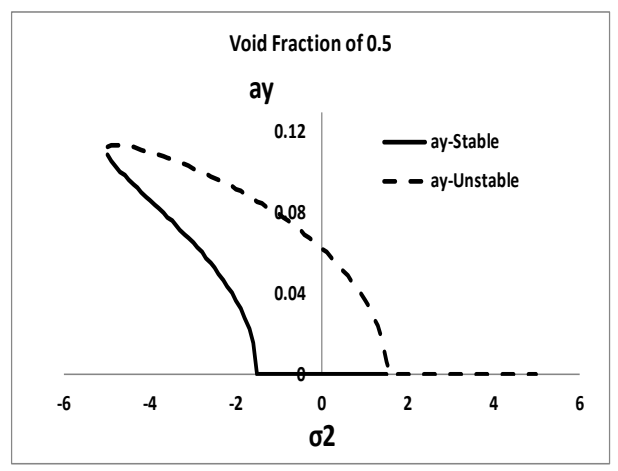

(f)

Fig. 2 Frequency response plots of the transverse parametric resonance of the second

\subsection{Results for $\omega$ close to $2 \lambda$ (Internal resonance condition)}

Figure 3 show that when the pulsation frequency is coincident with the transverse natural frequency, the axial response peaks at infinity with no vibrations in the transverse plane. Apparently due to the nonlinear internal coupling between the axes, the absence of the transverse oscillations when the axial peaks to infinity will aid in neutralizing the axial 
resonance peaks. The time traces of the tip motions for a perfectly tuned internal resonance with the pulsation frequency slightly detuned by 0.5 for all the void fractions as depicted in Figure 6 of Appendix 1 are observed to be with beating time series and dense phase plane orbits which fills up the phase plane. The spectra density plots exhibited four concentrated peak which is confirms a quasiperiodic motion with four dominating frequencies.

Pre-critical mixture velocity

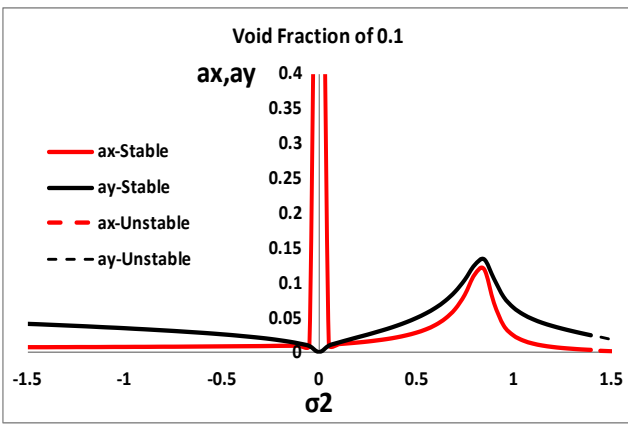

(a)

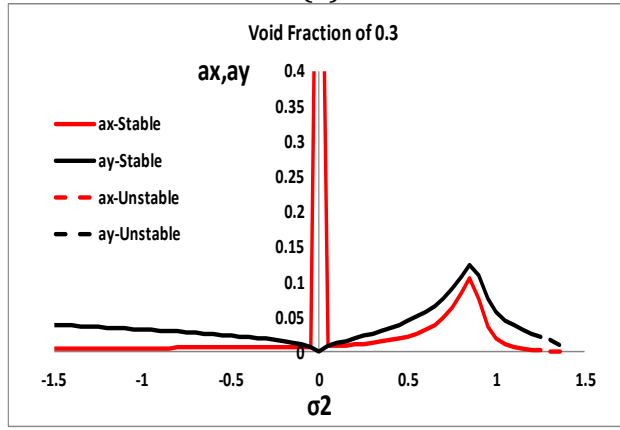

(c)

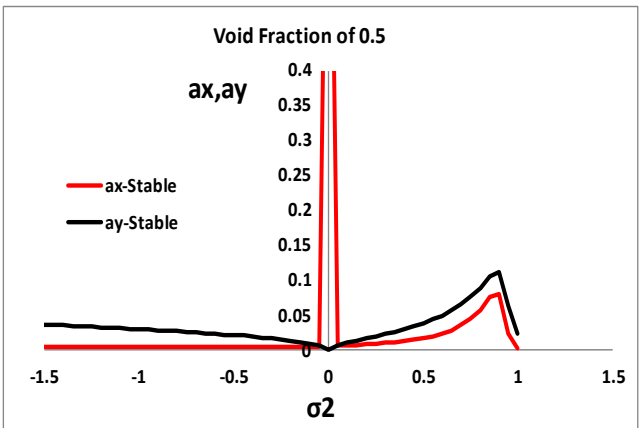

(e)

\section{Post-critical mixture velocity}

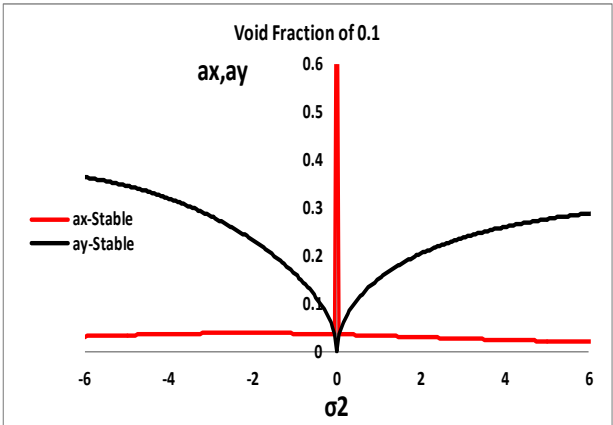

(b)

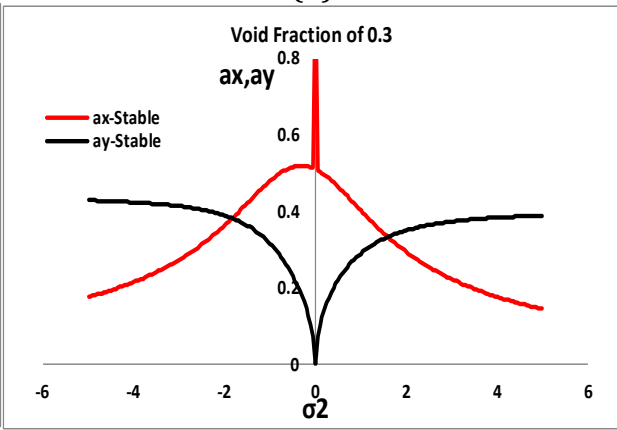

(d)

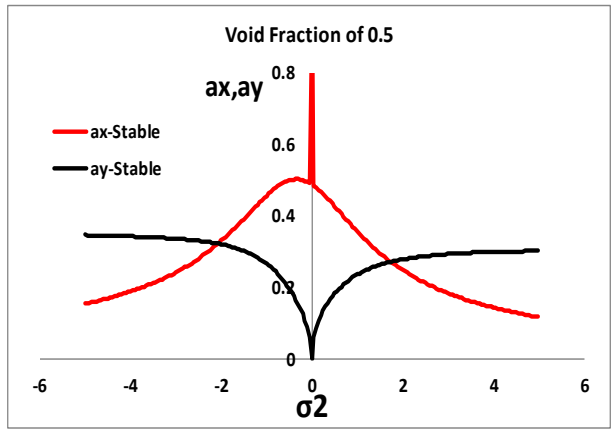

(f)

Fig. 2 Frequency response plots of the perfectly tuned $\left(\sigma_{\mathbf{1}}=\mathbf{0}\right)$ internal resonance of

\section{Conclusion}

This work advanced the knowledge of nonlinear dynamics of parametric forced vibrations of cantilever pipe with pulsating two-phase flow. Contrary to the dynamics of pulsating 
single-phase flow, the pulsating two-phase flow system presents three principal parametric resonance regimes. Obviously, these are exclusive characteristics of the planar dynamics of pulsating two-phase flow in pipes. With respect to practical applications, low void fractions would be enough for the analysis of two-phase flow systems. However, the 2:1 oscillatory pattern of the resonance response in the neighbourhood of the critical points motivated the extension of this analysis to flow conditions with high void fractions. In the absence of internal resonance, the frequency response exhibits softening nonlinear behaviour for the void fractions considered in this analysis. This is valid for pre- and postcritical flow conditions. At higher void fraction, the post-critical flow dynamics indicated continuous interchange between subcritical and supercritical pitchfork bifurcations. Meanwhile, in the presence of internal resonance, the system exhibits nonlinear antiresonance properties.

\section{Nomenclature}

$\begin{array}{ll}\varepsilon & \text { Dimensionless book-keeping parameter } \\ \mathrm{u} & \text { Displacement in pipe's axial direction }(\mathrm{m}) \\ \mathrm{v} & \text { Displacement in pipe's transverse direction }(\mathrm{m}) \\ \Omega_{1}, \Omega_{2} & \text { Pulsation frequencies for the phases } \\ \mu_{1}, \mu_{2} & \text { Pulsation amplitudes for the phases } \\ \mathrm{U}_{\mathrm{j}} & \text { Flow velocity of individual phases }(\mathrm{m} / \mathrm{s}) \\ \mathrm{M}_{\mathrm{j}} & \text { Flow mass of individual phases }(\mathrm{Kg}) \\ \mathrm{m}_{\mathrm{p}} & \text { Mass of pipe }(\mathrm{Kg}) \\ \mathrm{v} & \text { Poisson ratio } \\ \mathrm{E} & \text { Modulus of Elasticity }\left(\mathrm{N} / \mathrm{m}^{2}\right) \\ \mathrm{I} & \text { Mass moment of Inertia }\left(\mathrm{kgm}{ }^{2}\right) \\ \mathrm{L} & \text { Length of pipe }(\mathrm{m}) \\ \mathrm{V}_{\mathrm{T}} & \text { Mixture velocity }(\mathrm{m} / \mathrm{s}) \\ \mathrm{U}_{\mathrm{g}}, \mathrm{U}_{\mathrm{l}} & \text { Superficial velocities }(\mathrm{m} / \mathrm{s}) \\ \mathrm{S} & \text { Slip ratio } \\ v_{f} & \text { Void ratio } \\ \alpha & \text { Pipe linear expansivity }\left({ }^{0} \mathrm{C}^{-1}\right) \\ \Delta \mathrm{T} & \text { Temperature difference }\left({ }^{0} \mathrm{C}\right) \\ \rho_{\mathrm{g}}, \rho_{\mathrm{l}} & \left.\text { Densities (Kg/m }{ }^{3}\right) \\ \mathrm{t} & \text { Time (s) }\end{array}$


Appendix I: Time trace, Phase plot and spectral density plots of the pipe's tip transverse motions.
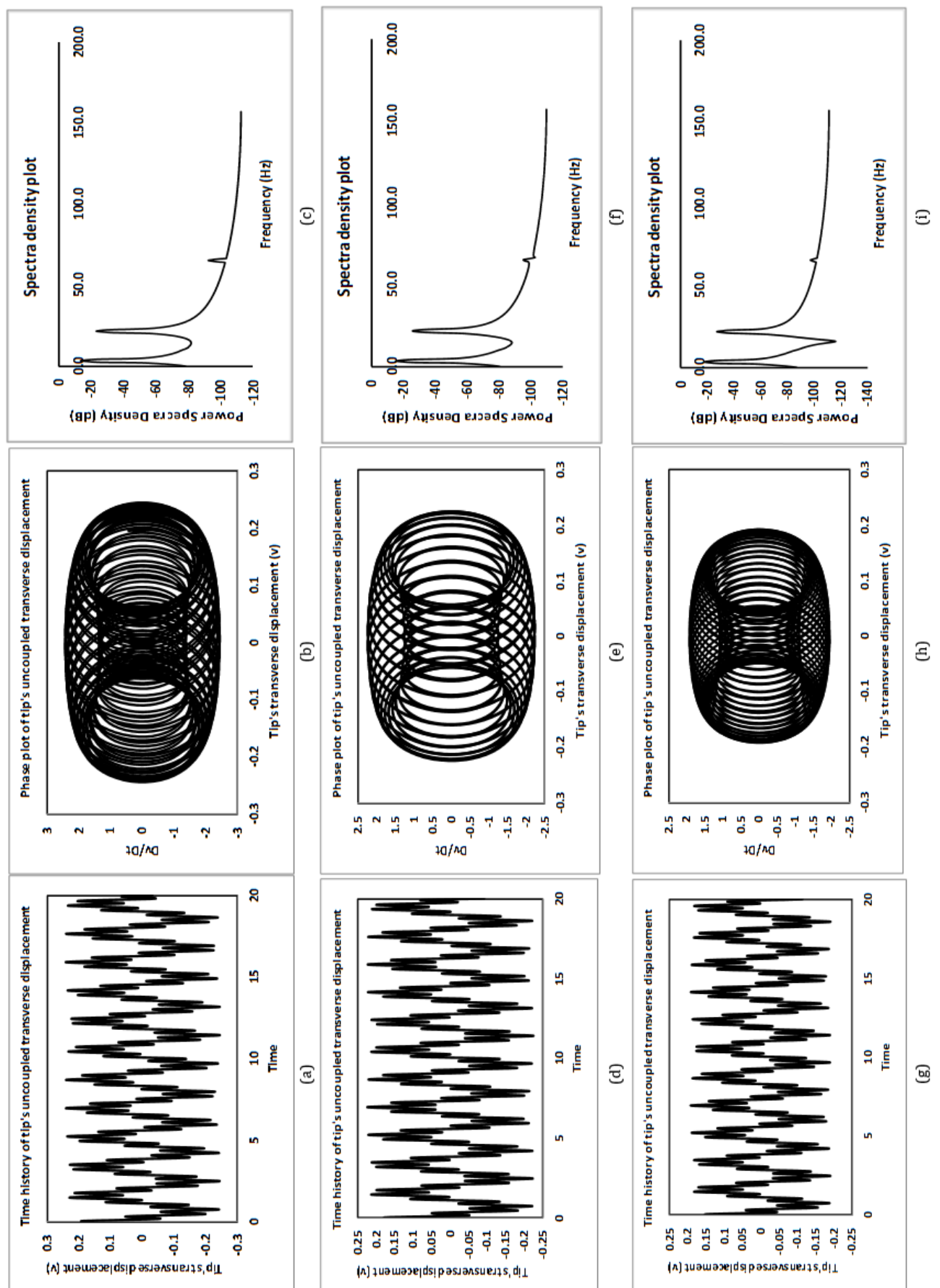

Fig. 4 Uncoupled time history, phase plots and spectral density plots of the pipe's tip transverse vibrations at pre-critical mixture velocity 

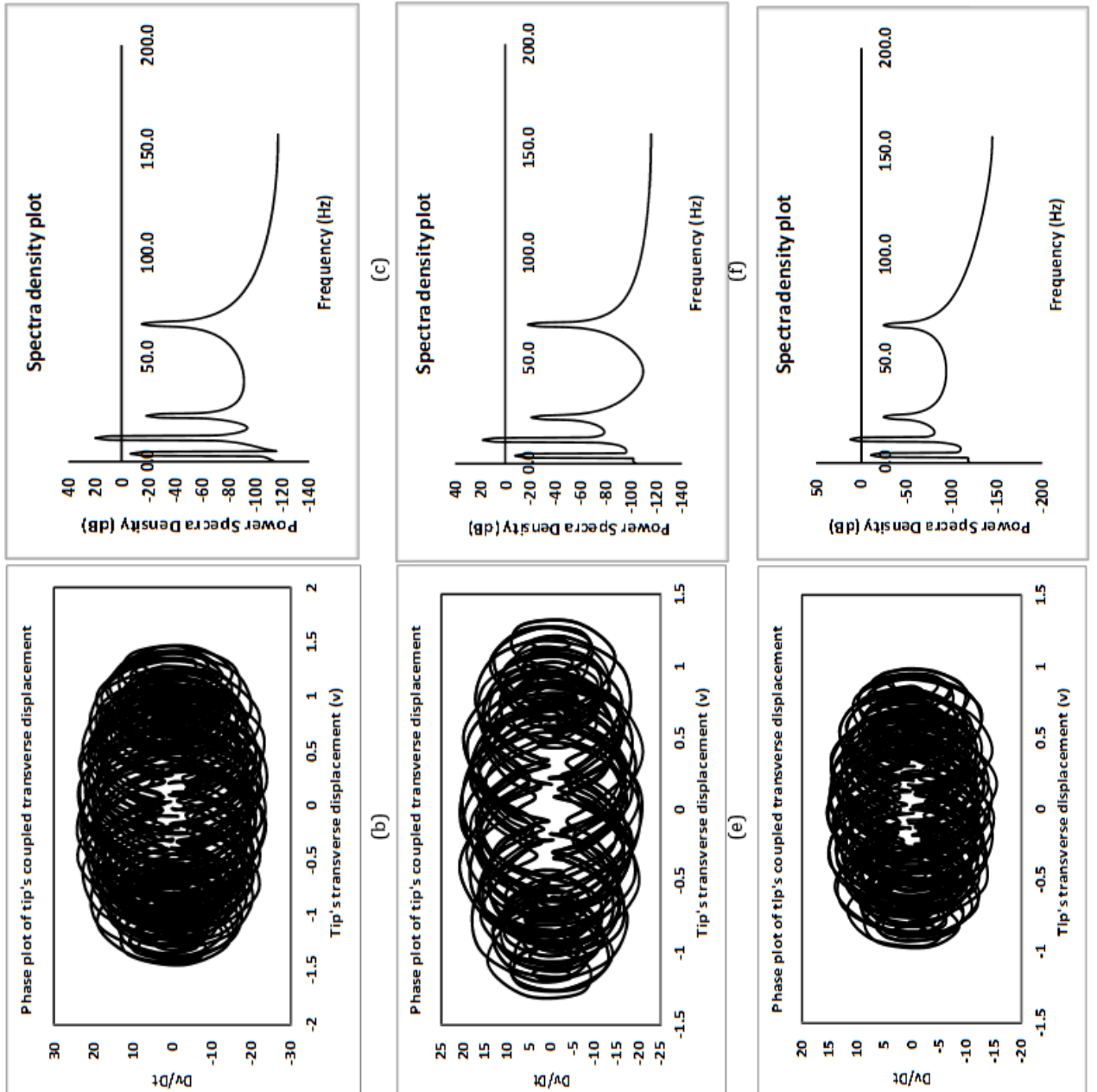

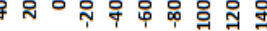
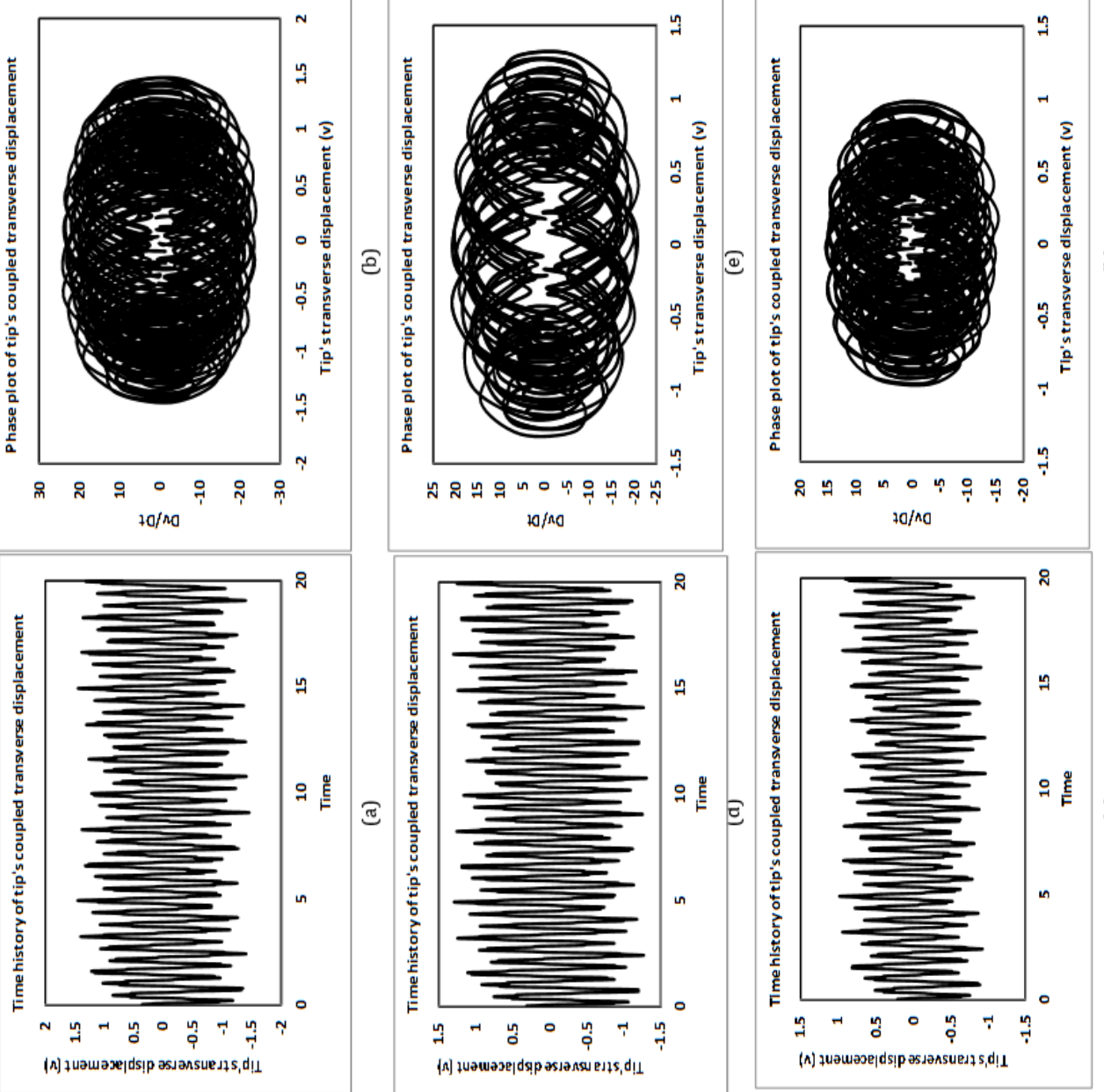

Fig. 5 Coupled time history, phase plots and spectral density plots of the pipe's tip transverse vibrations at pre-critical mixture velocity 


\section{Appendix II: Abbreviations}

$$
\begin{aligned}
& A 1=\left(C 21 \frac{\partial \phi(x)}{\partial x} \bar{U}_{1}+C 22 \frac{\partial \phi(x)}{\partial x} \bar{U}_{2}+2 \phi(x) \omega i\right) \\
& A 2=\left(\frac{\partial \eta(x)}{\partial x} \frac{\partial^{4} \eta(x)}{\partial x^{4}}+\frac{\partial^{2} \eta(x)}{\partial x^{2}} \frac{\partial^{3} \eta(x)}{\partial x^{3}}-C 6 \frac{\partial \eta(x)}{\partial x} \frac{\partial^{2} \eta(x)}{\partial x^{2}}\right) \varepsilon \sigma_{2} \\
& \mathrm{~B} 1=\left(C 21 \frac{\partial \eta(x)}{\partial x} \bar{U}_{1}+C 22 \frac{\partial \eta(x)}{\partial x} \bar{U}_{2}+2 \eta(x) \lambda i\right) \\
& \mathrm{B} 2=6\left(\frac{\partial \eta(x)}{\partial x}\right)^{2} \frac{\partial \overline{\eta(x)}}{\partial x}+2\left(\frac{\partial \eta(x)}{\partial x}\right)^{2} \frac{\partial^{4} \overline{\eta(x)}}{\partial x^{4}}+4 \frac{\partial \eta(x)}{\partial x} \frac{\partial \overline{\eta(x)}}{\partial x} \frac{\partial^{4} \eta(x)}{\partial x^{4}}+8 \frac{\partial \eta(x)}{\partial x} \frac{\partial^{2} \overline{\eta(x)}}{\partial x^{2}} \frac{\partial^{3} \eta(x)}{\partial x^{3}}+ \\
& 8 Y\left(T_{1}\right)^{2} \overline{Y\left(T_{1}\right)} \frac{\partial \eta(x)}{\partial x} \frac{\partial^{2} \eta(x)}{\partial x^{2}} \frac{\partial^{3} \eta(x)}{\partial x^{3}}-3 C 6 \frac{\partial \eta(x)}{\partial x} \frac{\partial \overline{\eta(x)}}{\partial x} \frac{\partial^{2} \eta(x)}{\partial x^{2}}+8 \frac{\partial \eta(x)}{\partial x} \frac{\partial^{2} \eta(x)}{\partial x^{2}} \frac{\partial^{3} \eta(x)}{\partial x^{3}}- \\
& \frac{3}{2} C 6\left(\frac{\partial \eta(x)}{\partial x}\right)^{2} \frac{\partial^{2} \eta(x)}{\partial x^{2}} \\
& \mathrm{~B} 3=\left(2 \frac{\partial \Phi(x)}{\partial x} \frac{\partial^{4} \overline{\eta(x)}}{\partial x^{4}}+\frac{\partial \overline{\eta(x)}}{\partial x} \frac{\partial^{4} \Phi(x)}{\partial x^{4}}+4 \frac{\partial^{2} \Phi(x)}{\partial x^{2}} \frac{\partial^{3} \overline{\eta(x)}}{\partial x^{3}}+3 \frac{\partial^{2} \overline{\eta(x)}}{\partial x^{2}} \frac{\partial^{3} \Phi(x)}{\partial x^{3}}-C 6 \frac{\partial \Phi(x)}{\partial x} \frac{\partial^{2} \overline{\eta(x)}}{\partial x^{2}}-\right. \\
& \text { C6 } \left.\frac{\partial \overline{\eta(x)}}{\partial x} \frac{\partial^{2} \Phi(x)}{\partial x^{2}}\right) \\
& \mathrm{B} 4=\left(\frac{1}{2}\left(C 22 \mu_{2} \frac{\partial \overline{\eta(x)}}{\partial x} \bar{U}_{2} \lambda\right)-\frac{1}{2}\left(C 42 \varepsilon \sigma_{2} \mu_{2} \frac{\partial \overline{\eta(x)}}{\partial x} \bar{U}_{2}\right)+a C 32 \mu_{2} \frac{\partial^{2} \overline{\eta(x)}}{\partial x^{2}} \bar{U}_{2}{ }^{2} i-\right. \\
& \text { C32 } \left.\mu_{2} \frac{\partial^{2} \overline{\eta(x)}}{\partial x^{2}} \bar{U}_{2}^{2} i\right) \\
& \mathrm{B} 5=\left(\frac{1}{2}\left(C 21 \mu_{1} \frac{\partial \overline{\eta(x)}}{\partial x} \bar{U}_{1} \lambda\right)-\frac{1}{2}\left(C 41 \varepsilon \sigma_{2} \mu_{1} \frac{\partial \overline{\eta(x)}}{\partial x} \bar{U}_{1}\right)+a C 31 \mu_{1} \frac{\partial^{2} \overline{\eta(x)}}{\partial x^{2}} \bar{U}_{1}^{2} i-\right. \\
& \left.C 31 \mu_{1} \frac{\partial^{2} \overline{\eta(x)}}{\partial x^{2}} \bar{U}_{1}{ }^{2} i\right)
\end{aligned}
$$




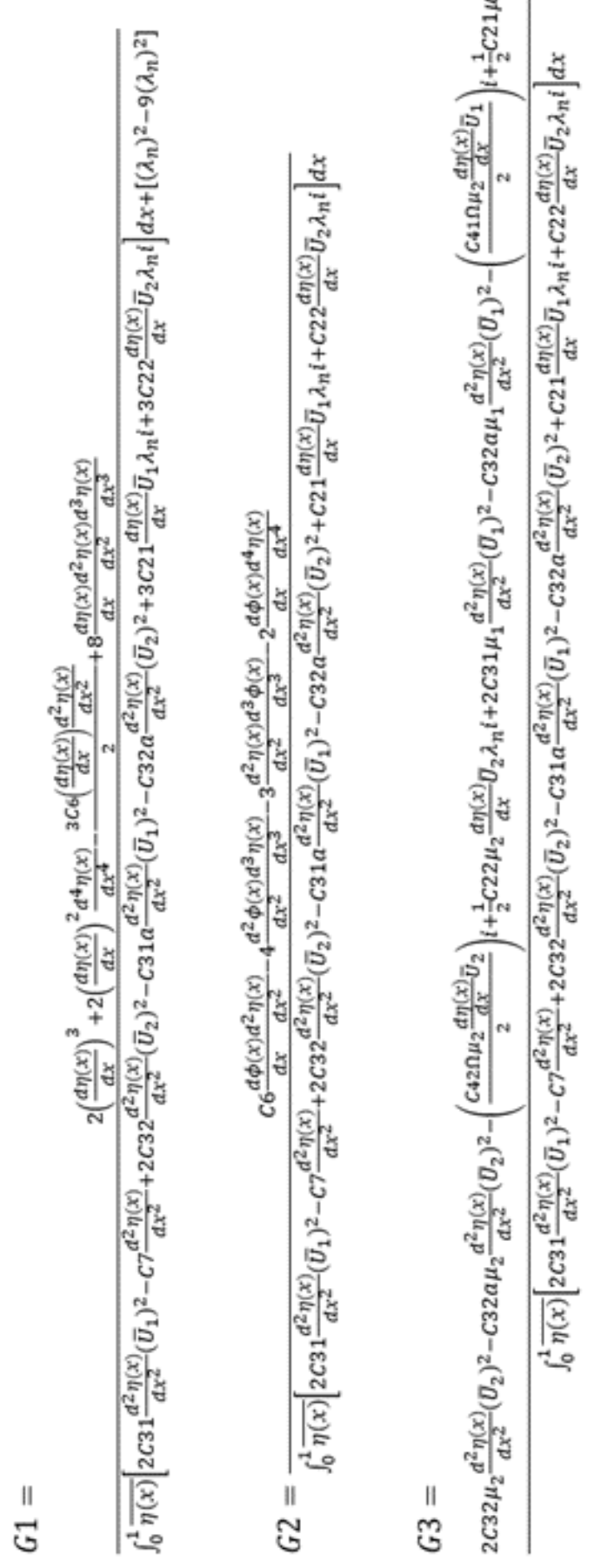




\section{References}

[1] Song R, Streit P. Design of the world's deepest hybrid riser system for cascade and chinook development. Offshore Technological Conference (OTC). 2011. https://doi.org/10.4043/21338-MS

[2] Chung J, Whitney AK, Loden WA. Nonlinear Transition Motion of Deep Ocean Mining Pipe. ASME J. Energy Resources Technology. 1981; 103: 2 - 10. https://doi.org/10.1115/1.3230811

[3] Gregory RW, Païdoussis MP. Unstable Oscillation of Tubular Cantilevers Conveying Fluid. I. Theory. Proceedings of the Royal Society of London Series A Mathematical and Physical Sciences. 1966; 293: 512 - 527. https://doi.org/10.1098/rspa.1966.0187

[4] Païdoussis MP, Issid NT. Dynamic Stability of Pipes Conveying Fluid. Journal of Sound and Vibration. 1974; 33(3): 267 - 294. https://doi.org/10.1016/S0022460X(74)80002-7

[5] Shilling R, Lou YK. An Experimental Study on the Dynamic Response of a Vertical Cantilever Pipe Conveying Fluid. Journal of Energy Resource Technology. 1980; 102(3): 129 - 135. https://doi.org/10.1115/1.3227862

[6] Semler C, Li GX, Païdoussis MP. The Nonlinear Equations of Motion of Pipes Conveying Fluid. Journal of Sound and Vibration. 1994; 169: 577 - 599. https://doi.org/10.1006/jsvi.1994.1035

[7] Ghayesh MH, Païdoussis MP, Amabili M. Nonlinear Dynamics of Cantilevered Extensible Pipes Conveying Fluid. Journal of Sound and Vibration. 2013; 332: 6405 - 6418. https://doi.org/10.1016/i.jsv.2013.06.026

[8] Ibrahim RA. Mechanics of Pipes Conveying Fluids-Part 1 Fundamental Studies. Journal of Pressure Vessel Technology. 2010: 113. https://doi.org/10.1115/1.4001271

[9] Łuczko J, Czerwiński A. Parametric Vibrations of Pipes Induced by Pulsating Flows in Hydraulic Systems. Journal of Theoretical and Applied Mechanics. 2014; 52(3): 719 730.

[10] Ginsberg JH. The Dynamic Stability of a Pipe Conveying a Pulsatile flow. International Journal of Engineering Science. 1973; 11: 1013 - 1024. https://doi.org/10.1016/00207225(73)90014-1

[11] Paidoussis MP, Issid NT. Experiments on Parametric Resonance of Pipes Containing Pulsatile Flow, Journal of Applied Mechanics, 1976; 43: 198 - 202. https://doi.org/10.1115/1.3423807

[12] Paidoussis MP, Sundararajan C. Parametric and combination resonances of a pipe conveying pulsating fluid. Journal of Applied Mechanics. 1975; 42: 780 - 784. https://doi.org/10.1115/1.3423705

[13] Semler C, Païdoussis MP. Nonlinear Analysis of the Parametric Resonance of a Planar Fluid-Conveying Cantilevered Pipe. Journal of Fluids and Structures. 1996; 10: 787 825. https://doi.org/10.1006/ifls.1996.0053

[14] Namachchivaya NS, Tien WM. Bifurcation Behaviour of Nonlinear Pipes Conveying Pulsating Flow. Journal of Fluids and Structures. 1989; 3: 609 - 629. https://doi.org/10.1016/S0889-9746(89)90157-6

[15] Panda LN, Kar RC. Nonlinear Dynamics of a Pipe Conveying Pulsating Fluid with Combination. Principal Parametric and Internal Resonances. Journal of Sound and Vibration. 2008; 309: 375 - 406. https://doi.org/10.1016/j.jsv.2007.05.023

[16] Liangqiang Z, Fangqi C, Yushu C. Stability and Bifurcation Analysis of a Pipe Conveying Pulsating Fluid with Combination Parametric and Internal Resonance. Mathematical and Computational Applications. 2016; 20: 200 - 216. https://doi.org/10.19029/mca$\underline{2015-017}$

[17] Oz HR, Boyaci H. Transverse Vibrations of Tensioned Pipes Conveying Fluid with Time-dependent Velocity. Journal of Sound and Vibration. 2000; 236(3): 259 - 276. https://doi.org/10.1006/jsvi.2000.2985 
[18] Oz HR. Nonlinear Vibrations and Stability Analysis of Tensioned Pipes Conveying Fluid with Variable Velocity. International Journal of Nonlinear Mechanics. 2001; 36: 1031 1039. https://doi.org/10.1016/S0020-7462(00)00065-2

[19] Singh K, Mallik AK. Parametric Instabilities of a Periodically Supported Pipe Conveying Fluid. Journal of Sound and Vibration. 1979; 62: 379 - 397. https://doi.org/10.1016/0022-460X(79)90631-X

[20] Yoshizawa M, Nao H, Hasegawa E, Tsujioka Y. Lateral Vibration of a Flexible Pipe Conveying Fluid with Pulsating Flow. Bulleting of Japan Society of Mechanical Engineers. 1986; 29: 2243 - 2250. https://doi.org/10.1299/jsme1958.29.2243

[21] Miwa S, Mori M, Hibiki T. Two-phase Flow Induced Vibration in Piping Systems. Progress in Nuclear Energy. 2015; 78: $270 \quad$ - 284. https://doi.org/10.1016/j.pnucene.2014.10.003

[22] Monette C, Pettigrew MJ. Fluidelastic Instability of Flexible Tubes Subjected to Two phase Internal Flow. Journal of Fluids and Structures. 2004; 19: 943 - 956. https://doi.org/10.1016/j.jfluidstructs.2004.06.003

[23] Adegoke AS, Oyediran AA. Natural Frequencies, Modes and Critical Velocities of Top Tensioned Cantilever Pipes Conveying Pressurized Steady Two-phase Flow Under Thermal Loading. Research on Engineering Structures and Materials Journal. 2018; 4(4): 297 - 323. https://doi.org/10.17515/resm2017.16en0301

[24] Adegoke AS, Oyediran AA. The Analysis of Nonlinear Vibrations of Top-Tensioned Cantilever Pipes Conveying Pressurized Steady Two-Phase Flow under Thermal Loading. Mathematical and Computational Applications. 2017; 22: 44. https://doi.org/10.3390/mca22040044

[25] Wang L, Yang Y, Li Y, Wang Y. Dynamic Behaviours of Horizontal gas-Liquid Pipes Subjected to Hydrodynamic Slug Flow: Modelling and experiments. International Journal of Pressure Vessels and Pipping. 2018; 161: 50 - 57. https://doi.org/10.1016/j.ijpvp.2018.02.005

[26] Enrique Ortiz-Vidal L, Castillo DG, Valverde Q. Theoretical Study on the Dynamic Behavior of Pipes Conveying Gas-Liquid Flow. MATEC Web of Conference. 2018; 148. https://doi.org/10.1051/matecconf/201814801004

[27] Woldesemayat MA, Ghajar AJ. Comparison of Void Fraction Correlations for Different Flow Patterns in Horizontal and Upward Inclined Pipes. International Journal of

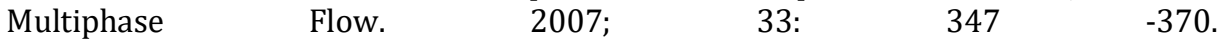
https://doi.org/10.1016/j.ijmultiphaseflow.2006.09.004 